\title{
Efficient Polishing of Aspheric Optics
}

\author{
Laboratory-Directed Research and Development Program \\ FY96 Final Report \\ 94-ERP-068
}

John S. Taylor, Mark A. Piscotty, Nhan Q. Nguyen, Charles S. Landram and Lawrence C. Ng

April 15, 1997

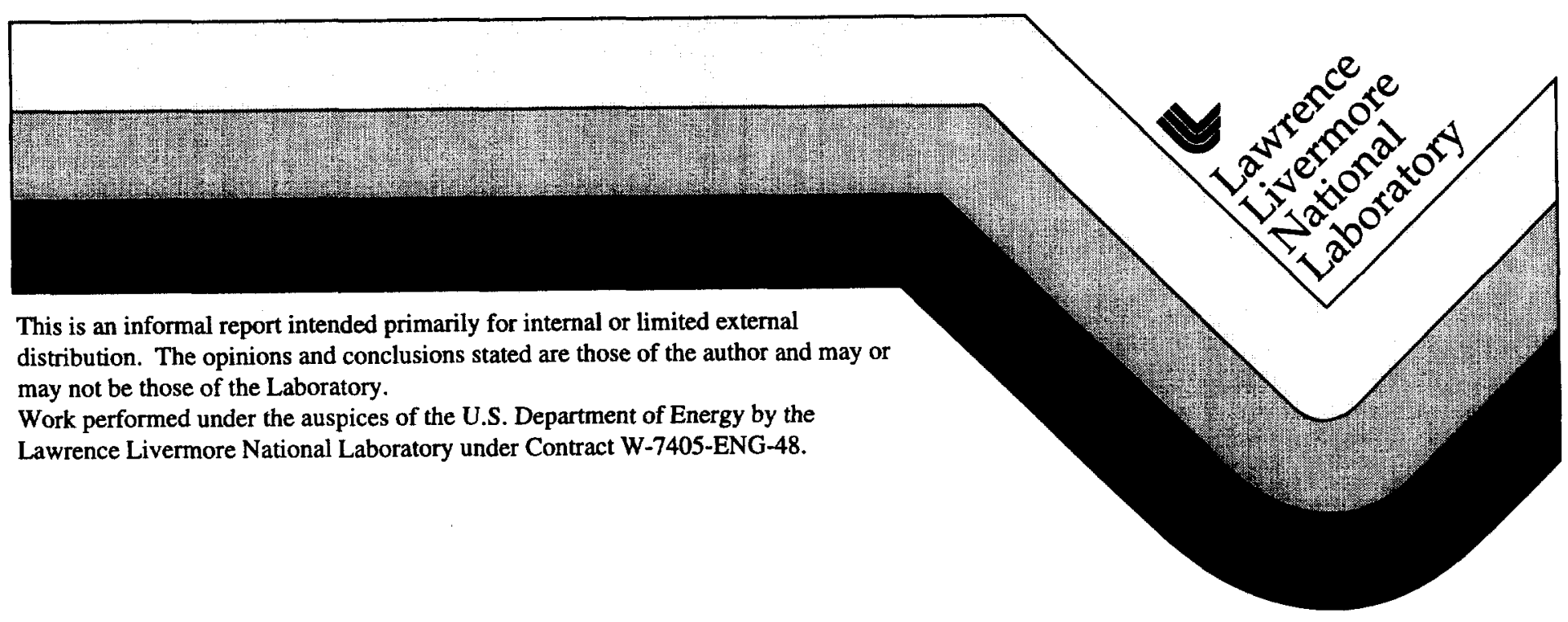




\section{DISCLAIMER}

Thls document was prepared as an account of work sponsored by an agency of the United States Government. Nelther the United States Goverament wor the University of Calfornia wor any of their employees, makes any warranty, express or Implled, or sssumes any legal liability or responsibility for the accuracy, completeness, or usefulness of any information, apparatus, product, or process diaclosed, or represents that its use would not infringe privately owned rtphts. Reference hereln to any specific commercial products, process, or service by trade name, trademark, manufacturer, or otherwise, does not necessarily constitute or imply its endorsement, recommendation, or favoriag by the United States Government or the Unlversity of Callfornla. The views and opinions of authors expressed herein do not necessarlly state or reflect those of the United States Government or the University of Calffornia, and shall not be used for advertising or product endorsement purposes.

Thls report has been reproduced directily from the best avallable copj.

Available to DOE and DOE contractors from the Onice of Scientific and Technical Information

P.O, Box 62, Oak Ridge, TN 37831

Prices avallable from (615) 576-8401, FTS 626-8401

Arallable to the public from the

National Technical Information Service

US. Department of Commerce

5285 Port Royal Rd.,

Springfield, VA 22161 


\title{
Laboratory-Directed Research and Development Program FY96 Final Report
}

\author{
Efficient Polishing of Aspheric Optics \\ 94-ERP-068
}

$\begin{array}{ll}\text { Principal Investigator: } & \text { John S. Taylor } \\ & \text { Manufacturing and Materials Engineering Division } \\ & \text { Lawrence Livermore National Laboratory } \\ & \text { Livermore, CA } 94551 \\ & 510-423-8227\end{array}$

\section{Co-Investigators:}

Mark A. Piscotty

Manufacturing and Materials

Engineering Division

510-422-2052

Nhan Q. Nguyen

Manufacturing and Materials

Engineering Division

$510-422-7458$
Charles S. Landram

Nuclear Test Engineering

Division

510-422-8569

Lawrence C. $\mathrm{Ng}$

Engineering Research Division 510-422-8741

\begin{abstract}
The objectives of this project are to develop, evaluate, and optimize novel designs for a polishing tool intended for ultra-precise figure corrections on aspheric optics with tolerances typical of those required for use in extreme ultraviolet (EUV) projection lithography. This work may lead to an enhanced US industrial capability for producing optics for EUV, $x$-ray and, other high precision applications. LLNL benefits from developments in computer-controlled polishing and the insertion of fluid mechanics modeling into the precision manufacturing area. Our accomplishments include the numerical estimation of the hydrodynamic shear stress distribution for a new polishing tool that directs and controls the interaction of an abrasive slurry with an optical surface. A key milestone is in establishing a correlation between the shear stress predicted using our fluid mechanics model and the observed removal footprint created by a prototype tool. In addition, we demonstrate the ability to remove $25 \mathrm{~nm}$ layers of optical glass in a manner qualitatively similar to macroscopic milling operations using a numericallycontrolled machine tool. Other accomplishments include the development of computer control software for directing the polishing tool and the construction of a polishing testbed.
\end{abstract}




\section{Motivation and Overview}

Technology for manufacturing surfaces to precision tolerances is critical to LLNL Programs such as ICF and EUV Lithography and is a significant contributor to the economic well-being of the US. The importance of this area is clearly illustrated by the $\$ 140 \mathrm{M}$ cost of optics for the National Ignition Facility and the estimated $\$ 350$ billion world-wide market for optical systems by the year $2030 .^{1}$ Importantly, the key to future reductions in semiconductor linewidths via projection lithography may be limited by the availability of highly accurate aspheric optics. ${ }^{2}$

The objective of this project was to apply unique LLNL talents to synthesize a novel polishing tool to reduce the cost of fabricating high quality aspheric optics. Small subaperture tools, such as the one described in this report, appear to be necessary for making very precise corrections to general aspheric contours and for removing higher-order refractive index errors in transmissive optics. We envision this tool being used to reduce errors on optics, whose initial errors are about $\lambda / 20$ $\mathrm{rms}(\lambda=633 \mathrm{~nm})$, to less than $\lambda / 1000 \mathrm{rms}$ for spatial wavelengths greater than about $2 \mathrm{~mm}$.

\section{Rationale}

Several new optical figuring technologies are being developed in the US and elsewhere to eliminate the poor repeatability and high cost associated with traditional pitch polishing. Ion beam figuring ${ }^{3}$ (IBF) and plasma-assisted chemical etching ${ }^{4}$ (PACE) both have controllable removal footprints that may be applicable for high accuracy figuring, but both require expensive vacuum systems and are applicable only to limited sets of materials. IBF, for example, has been very successful in accomplishing the final figure corrections of the Keck Telescope segments. ${ }^{5}$ Ductile-mode grinding ${ }^{6,7}$ shows promise as a deterministic shaping process for producing smooth damage-free surfaces, but has not yet produced highly accurate aspheric surfaces that do not require post-polishing, particularly in fused silica. Stressed-lap ${ }^{8}$ and stressed-part lapping ${ }^{9}$ are currently being used with good success for figuring large telescope optics, but have not been applied to the much smaller optics of interest here, especially with respect to the tight tolerances and relatively short spatial wavelengths of relevance to lithographic optics.

EEM $^{10,11}$ (originally called 'elastic emission machining'), flow polishing ${ }^{12}$, and float polishing ${ }^{13,14,15}$ are all non-contact polishing techniques that produce minimal subsurface damage. They apparently all utilize a similar fundamental material removal process: a fluid dynamic flow field carries a fine abrasive slurry to the optical surface, which transports away material by a sufficiently gentle mechanism that does not disrupt the structure of the surface layers. In one form or another, these processes are currently being used to prepare aspheric surfaces. In fact, Canon was reported to be developing EEM in Japan to figure aspheric surfaces in support of the AAMTRA consortium, which is pursuing soft $x$-ray lithography. ${ }^{16}$ 
After reviewing the strengths and weaknesses of the above optical finishing strategies, we have assembled a list of key desirable attributes of an ultra-precise figuring tool for aspheric optics as shown in Table 1.

Table 1. Requirements for an ideal ultra-precision figuring tool

\begin{tabular}{|l|l|}
\hline 1. & a well-controlled, temporally-stable removal footprint \\
\hline 2. & $\begin{array}{l}\text { a footprint function suitable for deconvolution calculations } \\
\text { (e.g. gaussian) for determining optimal traverse paths over the } \\
\text { optical surface }\end{array}$ \\
\hline 3. & $\begin{array}{l}\text { an adjustable footprint shape and removal rate for accommodating } \\
\text { different error profiles on a wide range of aspheric contours }\end{array}$ \\
\hline 4. & $\begin{array}{l}\text { a footprint function that can correct errors for key spatial } \\
\text { wavelengths without introducing significant errors in other } \\
\text { wavelength bands }\end{array}$ \\
\hline 5. & a process that can be used on a wide variety of materials \\
\hline 6. & a process that introduces little or no subsurface damage \\
\hline 7. & $\begin{array}{l}\text { removal rates that can be reduced to low levels and controlled to the } \\
\text { level of precision required for removing very small surface errors }\end{array}$ \\
\hline 8. & a system that does not require a large capital investment \\
\hline
\end{tabular}

Although many of the previously mentioned figuring methods are promising and are being used with varying degrees of success, they all lack one or more of the above criteria. In particular, EEM, which is being developed for precision figuring $(\lambda / 200 \mathrm{rms})$, has an ill-shaped footprint with minimal capability for in-process adjustment.

\section{Concept for Novel Non-Contact Polishing Tool}

We have designed a new non-contact polishing tool, ${ }^{17}$ shown in Fig. 1 , that has potential to meet all of the criteria listed in Table 1. A key aspect of this new tool concept is that it combines two different slurry flow geometries to provide flexibility in altering the shape of the removal footprint. By varying the relative contributions of the two components, we hypothesize that the footprint shape can be varied by controlling the relative strengths of the two independent flow regimes.

The characteristic flow contributions consist of a constrained radial jet flow (wall jet) and a rotationally-driven flow as shown in Figs. 1a and 1c. The radial flow is formed when slurry flowing axially through the shaft of the polishing tool impinges on the optical surface (stagnation flow) and is constrained to flow radially between the optical surface and the brim of the tool. The flow characteristics of this radial flow depend upon the precise shape of the brim, the location of the minimum gap between the brim and the surface, the traction force applied axially to the tool, and the slurry feed pressure. We postulate that material removal will be enhanced at locations of increased shear stress which should generally occur where the gap is minimized. 
The bottom surface of the brim may be azimuthally smooth, or it may have radial grooves or other features formed in it (Fig. 1b). As the tool rotates, it causes an azimuthal flow component to form, resulting in an enhanced shear stress at the optical surface. For the case of a smooth profile on the bottom the brim, a Couettelike flow forms, analogous to the shear flow caused by the relative sliding of two parallel plates. The example shown in Fig. 1c shows a tool that has radial grooves machined into the brim which may lead to the formation of a driven cavity flow. These driven cavities will give a vertical velocity component that may amplify the flux of fresh slurry particles to the surface, thus contributing to the shape of the removal footprint. Our estimates indicate that for the small radius tools under current study, the shear stress due to rotation will be dominated by the Couette flow region corresponding to the areas between the grooves. For moderate rotational speeds, the contribution from the cavities is relatively low for the specific geometry shown in Figure 1.

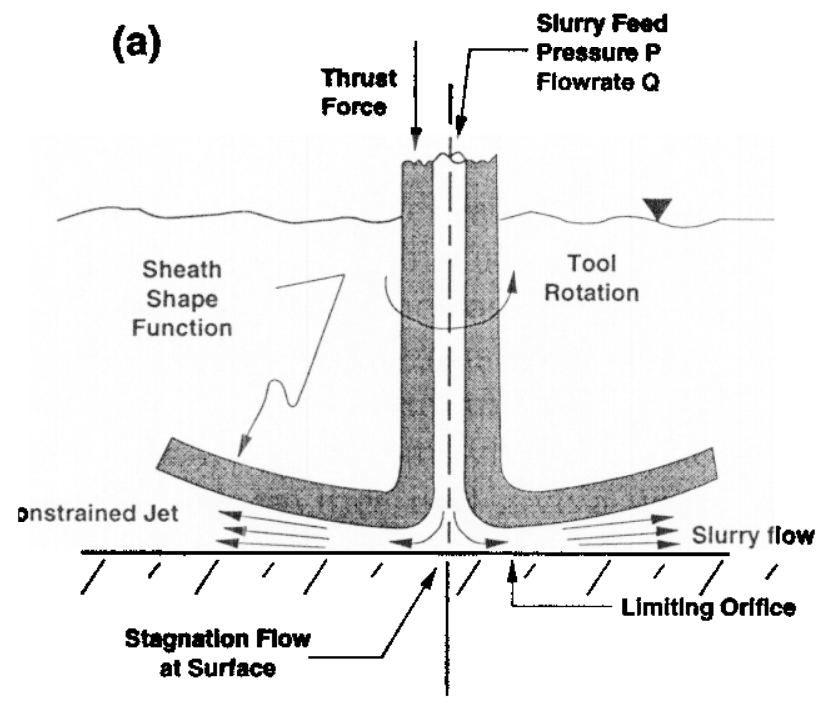

(b)

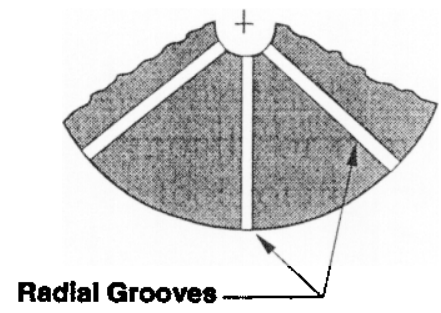

(c)

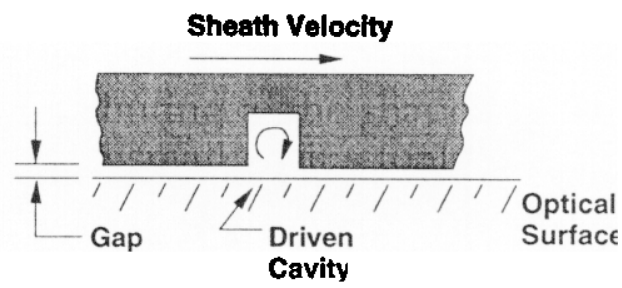

igure 1. Schematic of new polishing tool. (a) constrained radial jet flow; b) bottom view showing radial grooves; (c) azimuthal flow geometry Couette nd driven cavity flows.

This design has similarities to $\mathrm{EEM}^{10}$, flow polishing ${ }^{12}$, and float polishing ${ }^{13}$ as described in the literature. We feel that the constrained jet is more stable than the free impinging jet previously described, and provides the control by varying the ratio of the traction force to the supply pressure. The rotational flow may offer the Angstrom-level smoothing reported for float polishing, but could be applied to aspheric surfaces. The ability to control and change footprint geometry will provide a greater variety of useful footprint shapes than offered by EEM. 


\section{Experimental Set-up}

We have completed the design and construction of a polishing testbed, tool drive mechanism, and numerical control software to support our tool development experiments. The polishing testbed incorporates a precision machine base (Moore Jig Borer ${ }^{18}$ ), as depicted in Figure 2, which provides precision motion in the $\mathrm{x}$ and $\mathrm{y}$ axes for performing Cartesian scans of the optical surfaces with the polishing tool. A rotary table could be added in the future to enable $R-\theta$ scans.

The $x-y$ motions of the polishing testbed are computercontrolled using software written to support this project. These routines provide the interface that translates the user-provided commands of where and how fast to move the tool into the voltages necessary for driving the axis motors. A detailed description of the features of the numerical control software is provided in Appendix A.

A reed-flexure cantilever tool support mechanism for controlling tool-to-workpiece force is shown in

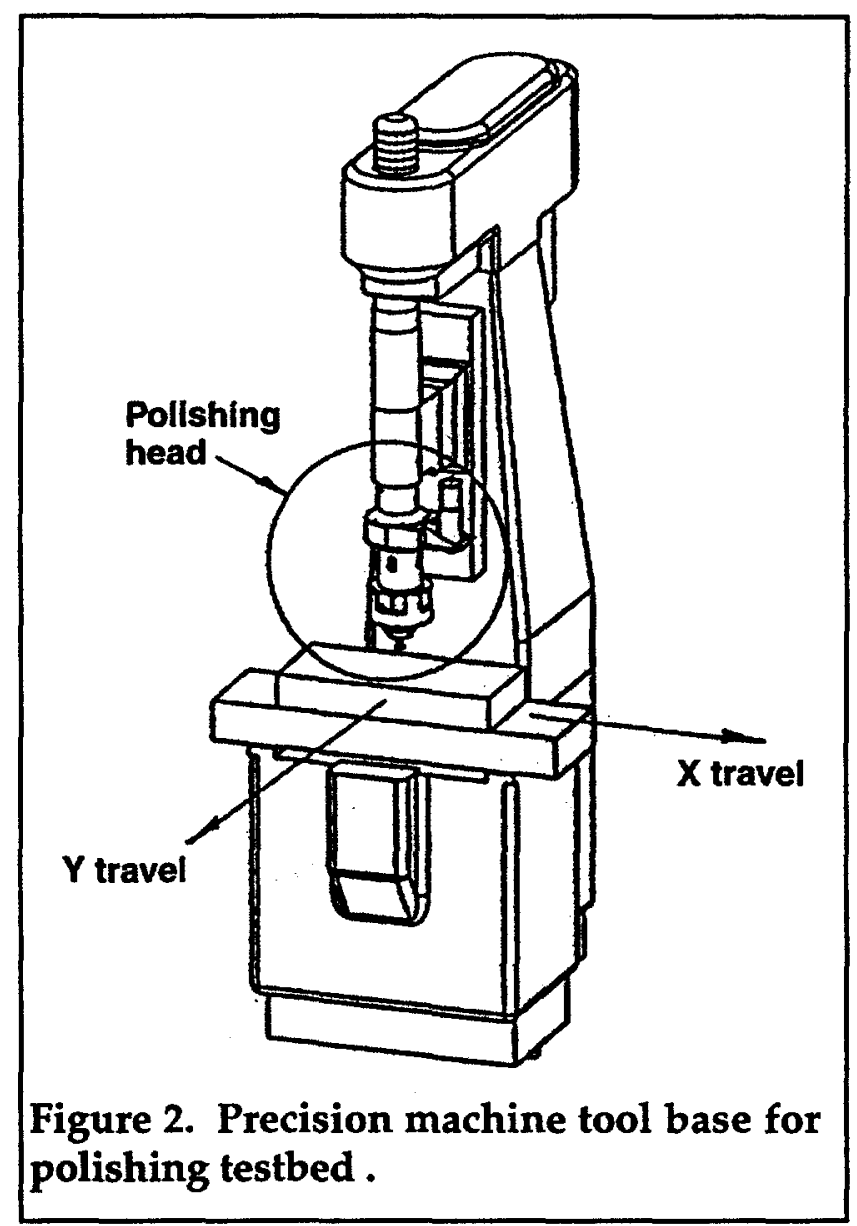

Figure 3. The spindle and motor are supported on opposite sides of a fulcrum point, and are coupled with an o-ring drive belt, shown below the baseplate. Auxiliary weights can be added to adjust the relative heights of the two sides, which is the method used for adjusting the tool-to-workpiece force. An air bearing spindle is used to support the tool in the radial and axial directions, as well as for coupling pressurized slurry to a feed hole in the tool shaft. Capacitance gauges are used to measure the angular motion of the flexure and the axial deflection of the air spindle.

Our typical procedure for using the reed-flexure set-up begins by lowering the (non-rotating) tool to the optical surface until contact is sensed by the capacitance gauge; this defines the neutral point as read on the "flexure" capacitance gauge. Slurry pressure can then be increased, which causes a force that deflects the tool away from the workpiece, after which tool rotation can be initiated. Depending on the nature of the test, more weight can then be added to limit the tool-workpiece gap height as sensed by the capacitance gauge. The force of the tool/slurry 


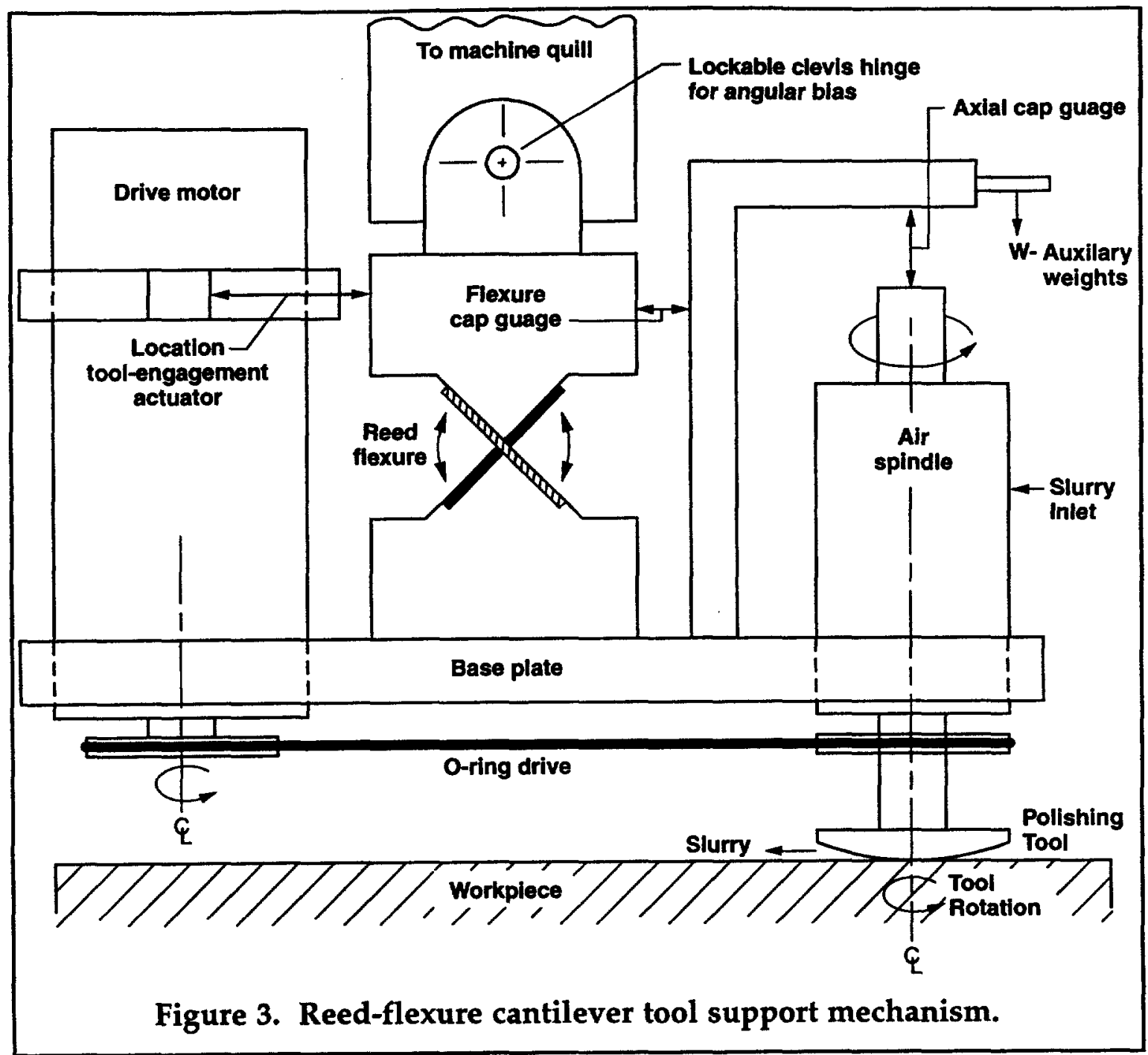

combination on the workpiece can be calculated from the combination of the added weights and the spring force provided by the flexures. For tests performed with no slurry flow, i.e. when slurry is only supplied from a source external to the tool, the slurry feed hole in the air spindle is plugged. Material removal begins as soon as either rotation and/or slurry pressure is added. To prevent erroneous "holes" being formed in the optical surface during the initial "set-down" or the final "lift-off", a small drive mechanism is used to engage/disengage the tool from the optical surface in synchronization with the initiation/conclusion of lateral scanning; this drive mechanism applies a force between the drive motor support and the upper flexure block at the location shown in Figure 3.

Our calculations and experiments indicate that an orbital tool motion offers a useful degree of freedom for controlling the shape of the polishing footprint. Thus, we designed and built a second tool drive system as shown in Figure 4. The orbital motion has the effect of taking the footprint and revolving it around a secondary axis, which results in a cycloid trace on the workpiece as the tool is scanned in the 
$x$-direction. In this design, the shaft is supported in the vertical direction by a metal bellows, labeled as "pad preload mechanism" in the figure. The vertical position of the shaft is set by using a precision regulator to adjust the air pressure within the bellows. The shaft can be laterally offset from the center of the system by a small orbital radius, which might range from $0-5 \mathrm{~mm}$. The shaft is driven about its centerline by motion supplied by the main machine tool spindle, where a flexible coupling is used to accommodate the misalignment due to the orbital offset. An auxiliary motor is shown that drives the orbital motion about a mechanical center determined by a set of rolling element bearings. Capacitance gauges (not shown) monitor the relative vertical motion of the shaft with respect to the machine tool column. In our initial experiments, we employed a commercial rotating union for coupling the slurry to the shaft. However, we found that the slurry contaminated the union's packing material, resulting in excessive frictional forces. Therefore, in subsequent experiments, we employed the air bearing coupling mentioned earlier, which was more tolerant of the slurry.

\section{Fluid Mechanics Modeling}

Our goal was to prepare a model of the shear stress distribution under the tool which could be used to optimize the shape of the removal footprint. We calculated the shear stress distribution under a specified tool contour with the hypothesis that the spatial distribution of material removal under the tool is strongly dependent upon the local shear stress. We indicate below that our observations of removal footprint shape support this hypothesis.

Our approach to modeling the shear stress distribution begins by dividing the flow into two regimes: a constrained radial jet and a rotational flow dominated by 
driven cavity effects. The Reynolds number (a strong indicator of shear stress) for each of these basic geometries was estimated and operating parameters were determined where the two flow regimes were approximately of equal strength. These operating conditions are within a reasonable range of pressures, flowrates and speeds. However a more-detailed analysis of driven cavity flows suggests that they are not sufficiently strong to enhance the shear stress distribution for the specific tools types investigated here. We conclude, however, that Couette flow, either in regions between grooves or for tools with no grooves, could play a significant role in contributing a rotational component to the shear stress. Groove structures may play an important role for other tool geometries, however, as they are observed to be important in float polishing. ${ }^{13}$

We identified the basic equations governing the flow of slurry from the supply orifice to the tool's outer diameter. The flow is divided into three characteristic regions: 1 ) a stagnation flow region in the near vicinity of the supply orifice, 2) a radial jet flow extending from the stagnation flow to the edge of the tool, and 3) an azimuthal flow region whose influence is concentrated toward the outer edge of the tool where the tangential velocity is greatest. Using these governing equations, we estimated the shear stress distribution for a tool that has a minimum radial flow gap located between the stagnation zone and the outside edge. The qualitative result of this calculation is shown in Fig. 5, which predicts a dramatic increase in shear stress at the location of the minimum flow gap. It is important that this prediction agrees with the experimentally-observed material removal distribution (Figure 9): material removal is dominated by a narrow ring located at the location of the minimum gap.

After observing the relative importance of the radial jet flow region in determining material removal, we refined our approach for calculating shear stress in the region. Our approach is diagrammed in Figure 6 and makes use of the Mangler transformation ${ }^{19}$ to convert the radial-flow geometry to a 2-D planar configuration. This enables us to employ the well-established Karman-Pohlhausen method for numerically solving the boundary layer equations for the shear

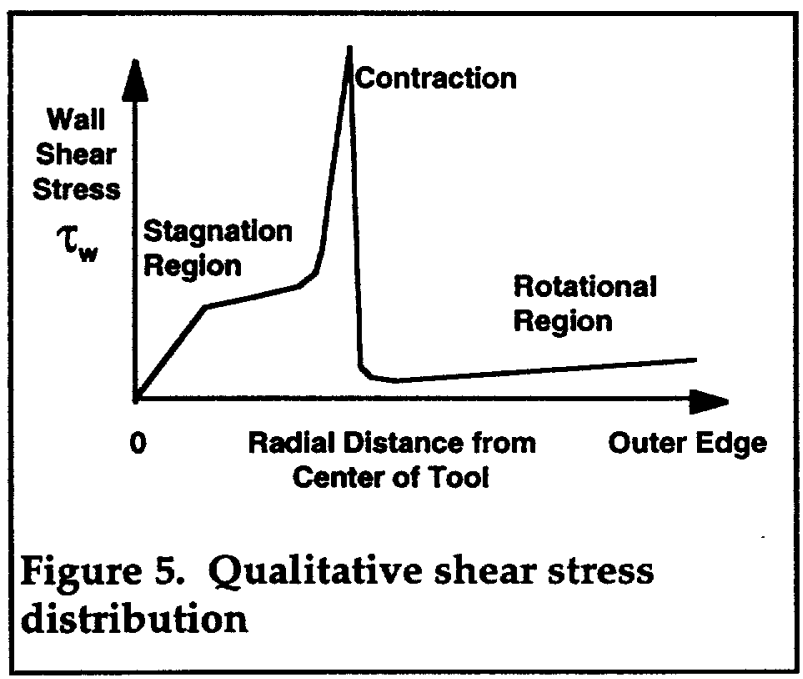
stress." We specify a tool contour and mass flowrate, solve the potential flow problem to obtain the pressure distribution, and then solve the boundary layer equations. The derivation of the equations for

In this analysis we assume that the radial jet flow and the rotational components can be estimated separately, i.e. no cross-coupling. We feel justified in making this assumption because the tangential motion provides a rather weak contribution to the overall fluid velocity. 
our approach, and a calculated shear stress profile for the radial flow component are presented in Appendix B. We propose that this procedure can be implemented iteratively to design a tool contour that optimizes the shear stress distribution.

\section{Convolution Modeling}

Removing material by traversing a footprint over a surface is a convolution process: ${ }^{20,21}$ the amount of material removed at a point is due to the removal contributions made for all of the locations taken by the tool. The ability to model this process depends on the accuracy of our knowledge of the footprint's shape and its motion over the optical surface. Conversely, a key requirement for a polishing operation to yield results that agree with a convolution prediction is for the shape and magnitude of the footprint to be temporally stable." As will be described in subsequent paragraphs, several aspects of convolution are important to this work.
1. Formulate boundary layer equations for axisymmetric incompressible radial flow (Can be solved for shear stress)

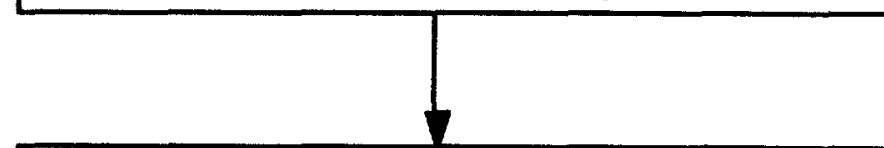

2. Transform radlally-dependent boundary layer
equations into planar $2-\mathrm{D}$ equations using Mangler Transformation
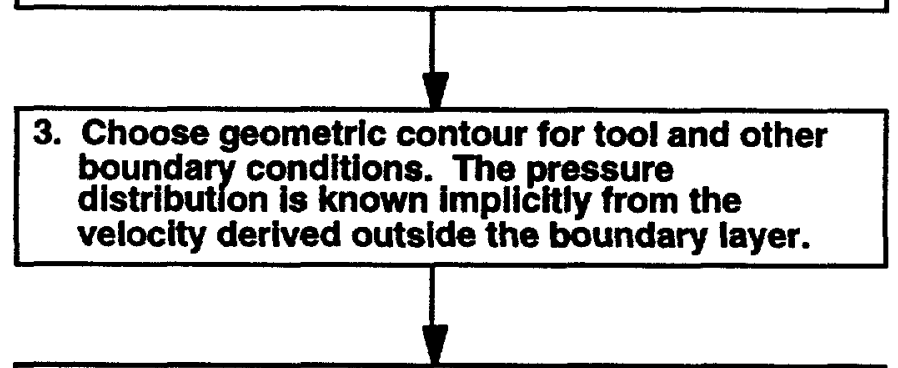

4. Solve 2-D boundary layer equations using Karman-Pohlhausen (KP) method to obtain 2-D shear stress distribution

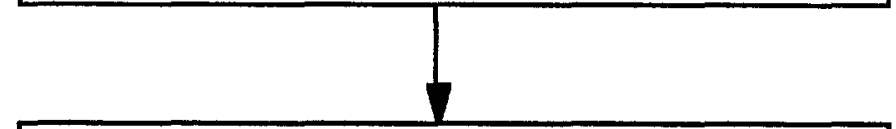

5. Apply inverse Mangler transformation to get axisymmetric, radial wall shear stress

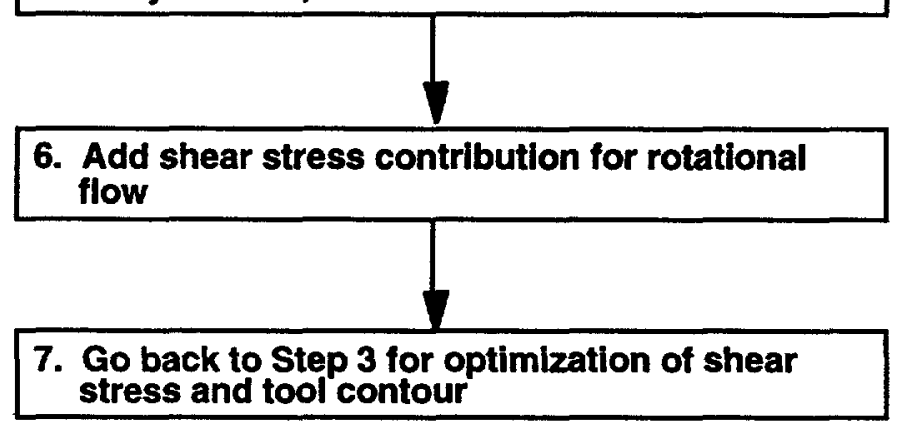

Figure 6. Diagram of modeling approach for calculating the shear stress distribution under the polishing tool.

One key application of convolution is the prediction of the distribution of material removal for a polishing (figuring) operation; this is the "forward" problem which is a direct convolution integral. This involves specifying the path that the tool takes over the surface, the shape of its removal footprint, and the time that the

"One of the limitations to traditional pitch polishing is that the pitch "flows" and thus its removal characteristics will change during the polishing operation. 
tool dwells at all locations along the path." The magnitude of material removal is predicted using an empirical conversion relating dwell time and mass removed. We have implemented numerical routines for accomplishing this prediction using Matlab $^{22}$, which are important for estimating tool performance when polishing optical contours. We have also employed the convolution calculation to predict results for short polishing tests, such as scanning a tool in straight line, or orbiting it about a secondary axis.

Figure 7a shows the 2-D profile of a conceptual polishing footprint that has depression in the center. This footprint is an approximation to a class of footprints produced by a prototype polishing tool, where minimal shear stress is created at the center. If this footprint is orbited about a secondary axis, a time-averaged footprint (Figure $7 b$ ) is obtained where the central depression is eliminated. This footprint is calculated as a simple convolution of the initial footprint about a circular path. Similarly, if the orbital footprint (Figure $7 \mathrm{~b}$ ) is traversed in a straight line, the convolved shape of the stripe shown in Figure 7c shows a gaussian-like character.

The plot in Figure 8a,b shows the residual errors estimated from simple parallel scanning by a footprint with a depression in the center, such as the one in
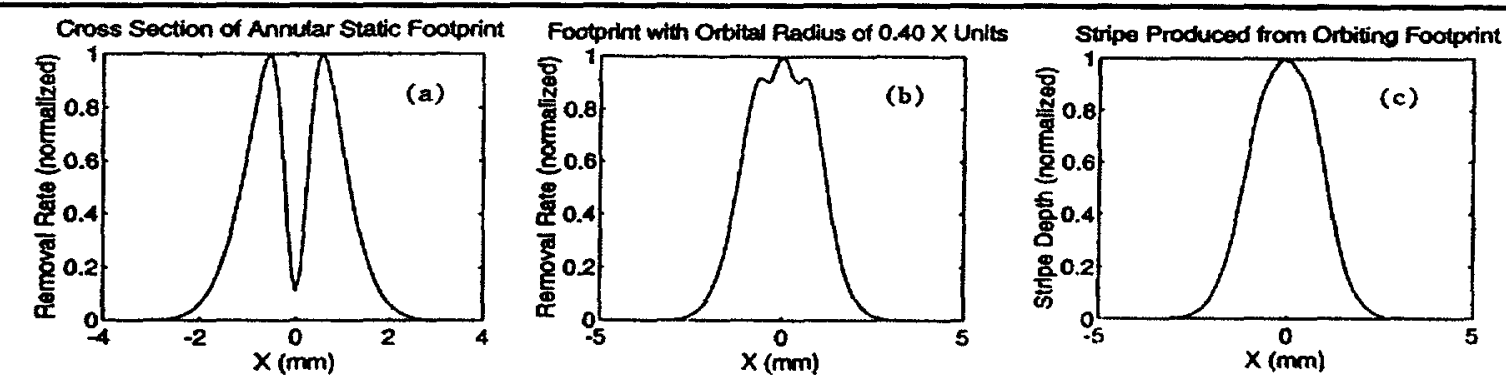

Figure 7. a) Example of footprint function comprised of summed gaussians; b) estimated footprint formed by orbiting the footprint in (a) about an auxiliary axis; c) gaussian-like profile produced b y scanning (convolving) this footprint along a linear axis.

Figure 7a. Figure $8 \mathrm{c}$,d shows the same scan, but with the footprint generated using the orbital rotation from Figure $7 \mathrm{~b}$. This simple convolution simulation illustrates that orbital rotation can lead to improvements in footprint shapes and in net removal profiles. We have also performed more detailed calculations that model the cycloid pattern traced out by an orbital rotation, and have been able to predict residual waviness on the surface that is created if inappropriate rotational speeds are employed.

Perhaps the most important convolution issue is the calculation of the optimal distribution of dwell times along a path, in order to optimize the

"One might alternatively specify the velocities taken by the tool as it traverses along its path. 
Material Removal Using Non-Orbiting Tool

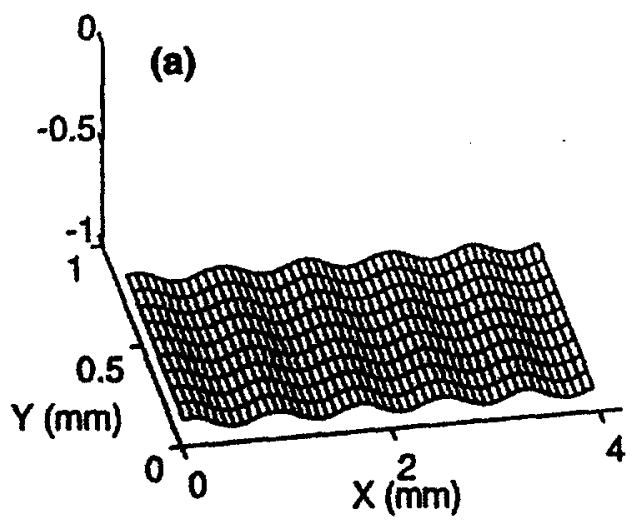

RMS surtace varlations $2.0 \%$ of mean depth

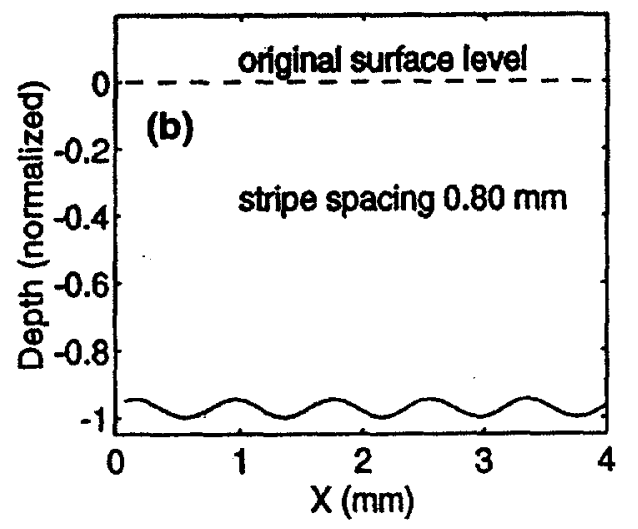

Material Removal Using Orbiting Tool

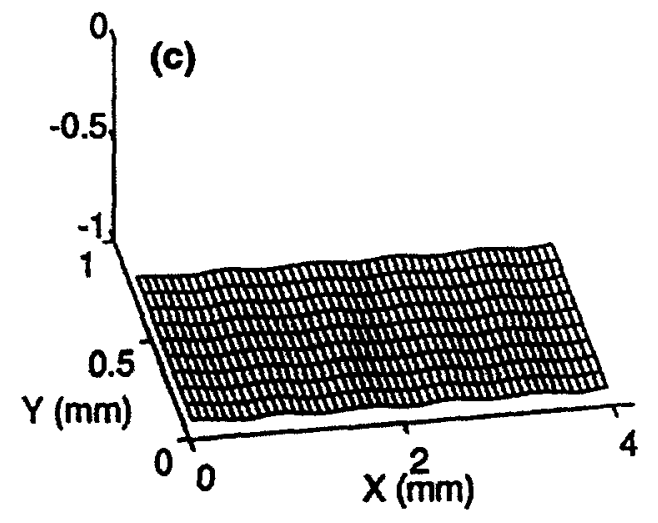

RMS surface variations $0.6 \%$ of mean depth

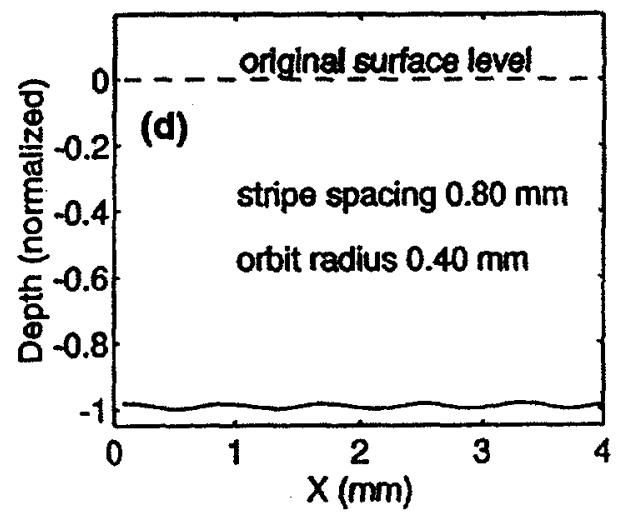

Figure 8. a) Convolution simulation using a tool footprint with a central depression (see Fig. 7); b) 2-D profile across scanned area showing that the residual error is $2 \%$ of the mean removal depth; $c, d)$ simulation with orbital motion; the error is reduced to $0.6 \%$ of the mean depth.

combination of residual errors and the processing time; this is the basis for the machine tool scanning instructions. This process is referred to as the deconvolution or the "inverse" problem and is typically more numerically difficult than the forward problem.

During this project, we have assembled a preliminary algorithm using Matlab that calculates the optimal set of dwell-times for a polishing tool for removing a given error profile. Although this software is still in development, it appears to provide a robust solution in the presence of noise in measured surface error profiles, as might be encountered for the very small errors of relevance to this project. Further work is required to demonstrate that these algorithms can address the complete family of errors that might exist on an actual surface, and in applying 
constraints as to the allowable velocities and accelerations in the tool's lateral motion.

\section{Summary of Experimental Results}

The goal of this section is to give a brief review of the types of removal experiments that were accomplished using our polishing testbed. The combination of $\mathrm{CNC}$ machine tool and precision polishing tool provide the ability to remove material in precise patterns with a flexibility similar to macroscopic milling operations, yet with submicron depths of cut on brittle work materials. In fact, many of our experiments yielded removal rates that produced individual features with removal depths less than $25 \mathrm{~nm}$ (1 microinch) and a control resolution sufficient to enable accurate contouring of surfaces.

A type of experiment that proved valuable was the generation of removal stripes, caused by a 1-D traversal of a tool (2-D removal footprint) over the surface. This traversal produces a trough, referred to here as a stripe, that is the convolution of the footprint shape over a linear sequence of dwell locations. Creating and measuring stripes was more useful than observing individual static footprints made using no lateral motion because static footprints were often confounded by the details of how the polishing test was initiated. For example, the repeatability of a footprint could be easily assessed by profiling across removal stripes created under nominally identical operating conditions, while measurements of static footprints often showed poor repeatability not attributable to tool variations.

An important experiment, where the results are illustrated using a removal stripe, was to qualitatively validate the hypothesis that material removal correlates with shear stress. For the tool geometry considered in Figure 1, the slurry has a stagnation flow in the central section of the tool near the orifice, and then flows radially as a constrained jet. Our prediction for this particular geometry is that the shear stress, and thus removal rate, will be negligible in the central section of the footprint, and then reach a sharp maximum near the limiting minimum flow orifice formed by the brim of the tool and the workpiece; this was shown qualitatively in Figure 5 and results in a prediction of an annular removal footprint.

Figure 9 shows an example of a stripe created with a tool that has an annular footprint where the removal is dominated by the constrained radial jet flow. Note that the removal has a rather steep increase/decrease at a radius which corresponds to the location of the minimal flow area. This stripe was made by three consecutive scans over the same area to increase the depth and facilitate measurement. The stripe has a depth of about 4-6 microinches $(100-150 \mathrm{~nm})$ at the edges and about 1 microinch $(25 \mathrm{~nm})$ in the center. The material removal rate for this test is estimated as $0.0016 \mathrm{~mm}^{3} / \mathrm{min}$. Subsequent tool designs were able to produce stripes

\footnotetext{
"For the polishing operations described in this report, material is not cut in the sense implied for macroscopic milling of ductile metals. Nevertheless, the appearance of a removal profile caused by the traversal of a polishing tool is analogous to the traversal of a milling cutter.
} 
with either a near 'top-hat' shape or with a smoother falloff in the vicinity of the radial jet orifice. Also, the addition of an orbital motion about a secondary axis provides a significant flexibility in changing the shape of the footprint.

Figure 10 shows a profile of a removal stripe generated with a tool that emphasizes rotational motion, as opposed to jet flow. This stripe has a

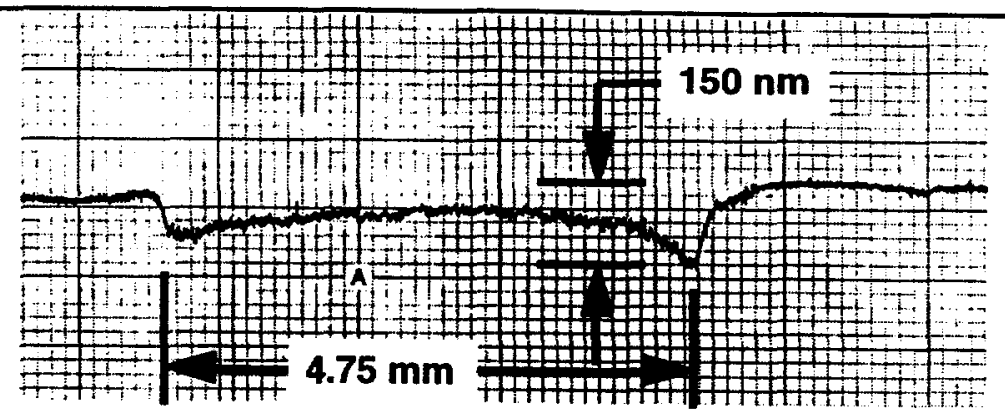

Figure 9. Depth profile across a removal stripe created by three passes of a prototype polishing tool; the work material is BK7 glass and the polishing agent was colloidal silica in a $\mathrm{pH} 10$ carrier; measured with the Federal Surfanalyzer. ${ }^{23}$

gaussian-like shape and

contrasts with the annular/top hat class of stripe shapes, such as that shown in the previous figure. This type of tool was more compatible with narrower removal features than the jet dominated tools.

In Figure 11 is shown a square geometric feature produced with the tool that produced the stripe in Figure 10. This feature is essentially a uniform level of removal over about a $5 \mathrm{~mm} \times 5 \mathrm{~mm}$ area with a depth of about $80 \mathrm{~nm}$. The tool traversed into the square from the stripe in the lower left corner, and exited via the stripe in the upper right corner. The square was created by raster scanning the tool, with a 'y-motion' jog at the end of each traverse. The light

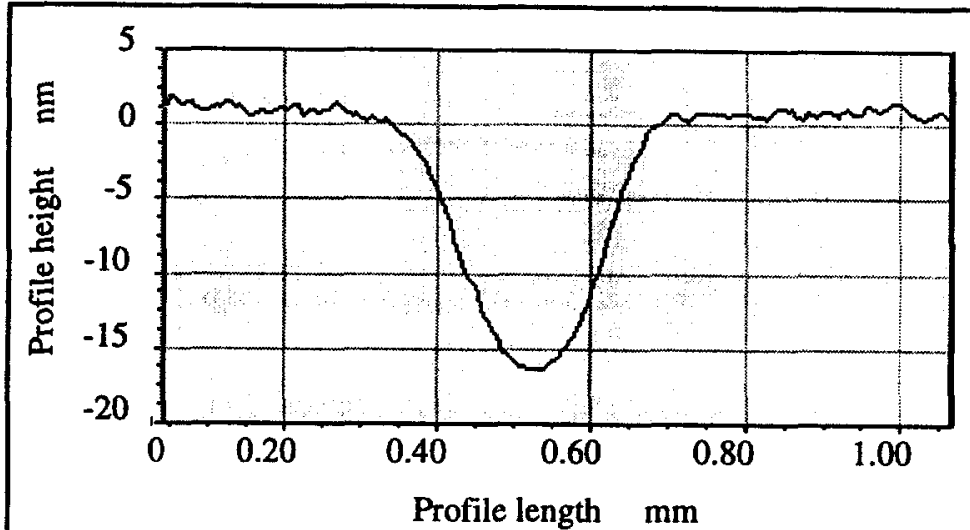

Figure 10. Depth profile of removal stripe from a single pass of a prototype polishing tool that emphasizes tool rotation; measured using a WYKO NT 2000 white light interferometric microscope. ${ }^{24}$ border around the edge of the square and stripes indicates the shoulder of the edge of the tool. The subtle ripple at the bottom of the stripes and individual raster scans is caused by a non-trueness in the motion of the tool, resulting in somewhat higher polishing forces once-per-revolution of the tool. A height profile measured horizontally across the square is shown in Figure 12. Longer range waviness inside the square is attributed to $x-y$ scanning motion errors due to a "tuning" problem in the servo-amplifier system. 
Surface Stutistics:

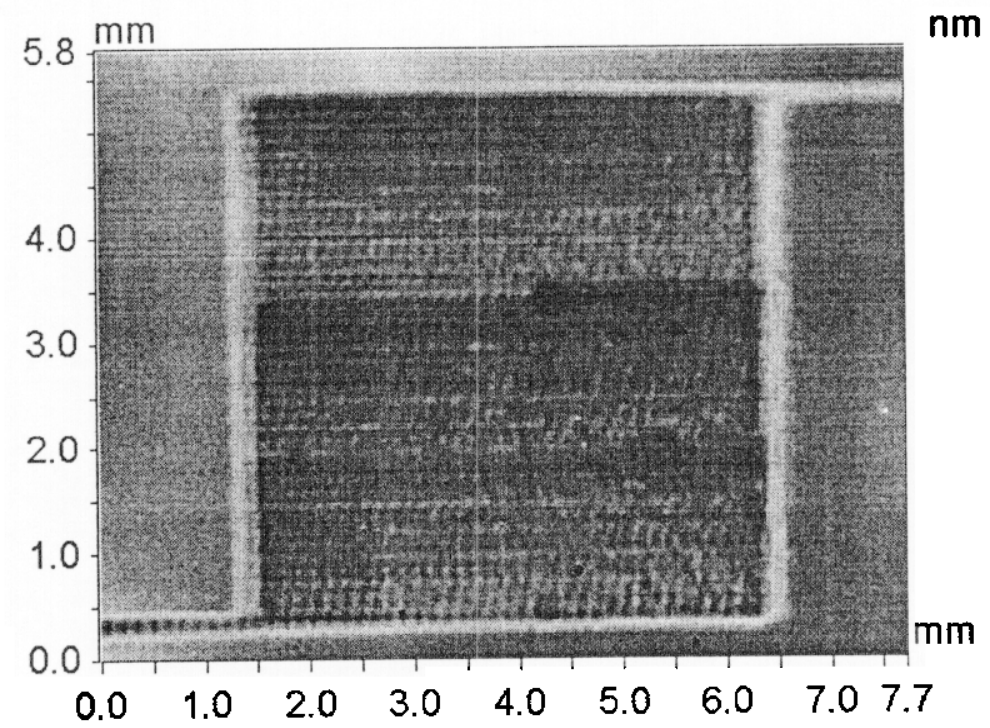

42.722

Ra: $11.89 \mathrm{~nm}$

Rq: $12.75 \mathrm{~mm}$

Rz: $62.01 \mathrm{~mm}$

Rt: $79.49 \mathrm{~nm}$

Set-up Parameters:

Size: $736 \times 471$

Sarmpling: 10.51 um

Processed Options:

Terms Removed:

Cylinder \& Tilt

Filtering:

fone

25.000

10.000

$-5.000$

$-20.000$

$-36.769$

gure 11. Example of a geometric feature polished (milled) into a BK7 lbstrate using a tool that emphasizes rotational removal mode; data taken ing a WYKO white light interferometric microscope.

The smoothness of the regions polished by small tools depends upon machine motion parameters, as well as the details of the material removal process. For example, if the spacing between neighboring raster scans is too large, the resulting surface texture is dominated by a ripple pattern, analogous to feedmarks observed in traditional machining operations. The surface profile shown in Figure 13 shows a smooth ripple pattern with approximately a $10 \mathrm{~nm}$ peakto-valley ripple that resulted from too coarse a spacing between neighboring raster lines. Our convolution

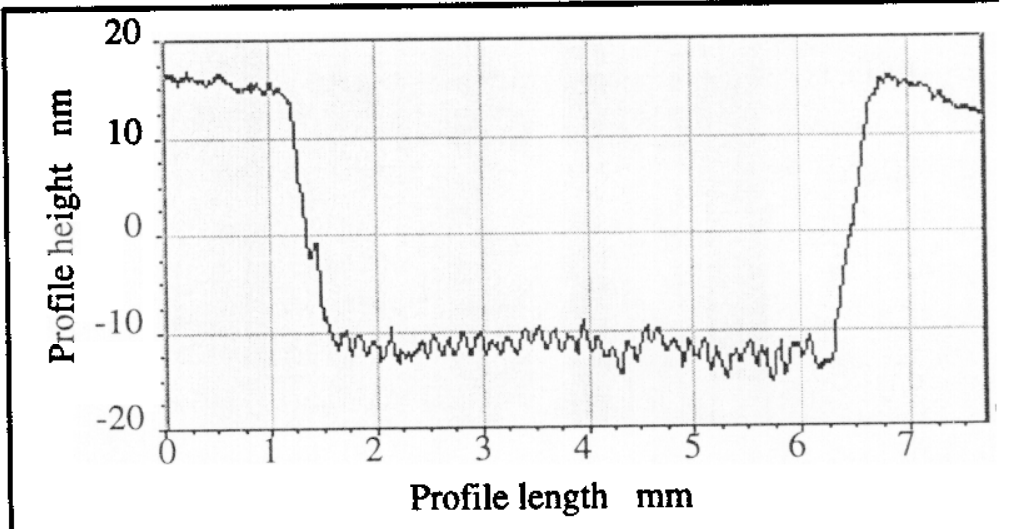

Figure 12. Horizontal profile of the square removal patch shown in Figure 11; measured with WYKO white light interferometric microscope. modeling routine is useful for predicting the magnitude of waviness for proposed tool scanning parameters. 


\section{Conclusions and} Recommendations

We have designed and tested a new type of polishing tool where the shape of the tool's removal footprint can be modified by varying the relative contributions from rotational motion and slurry jet flow. We hypothesize that material removal is largely determined by the distribution of fluid shear stress for this class of noncontact polishing tools. Fluid mechanics equations have been derived that predict the shear stress for the case of constrained radial jet flow under the tool. We observe qualitative agreement between the predicted shear stress distribution and the measured removal distribution for a removal footprint dominated by radial jet flow. Algorithms have been assembled to model the convolution of the tool footprint with the prescribed path and velocity profile (dwell functions) of the tool.

A polishing testbed has been constructed using a computer-numerically controlled machine tool and specially designed mechanisms that provide motion control and slurry to the tool. Computer control software was prepared to traverse the tool over the optical surface with a distribution of speeds determined from the convolution model.

A number of experiments have been performed that illustrate the range of footprint (or stripe) shapes that can be created using the tools described in this report. Tools that emphasize radial jet flow are shown to provide annular removal footprints; tools that provide rotational motion without being dominated by jet flow are shown to provide more gaussian-shaped removal profiles. The combination of these polishing tools with CNC machine tools offers the ability to create complex removal patterns on glass that have a depth of $\mathrm{O}(1-100 \mathrm{~nm})$. 
One problem that contributed to unwanted waviness in our polished surfaces is a tuning problem with the machine tool's servo-amplifier system. We recommend repairing or replacing this unit prior to further experimentation.

The polishing testbed is currently capable of scanning a tool over a flat surface for performing figure modifications on flat windows and mirrors. For curved surfaces, vertical motion is required for maintaining tool-to-workpiece contact pressure. In addition, a tilt stage (goniometer) should be incorporated for maintaining the tool axis normal to the workpiece surface.

\section{Acknowledgments}

The authors wish to extend their appreciation to several individuals that made important contributions during this project. We are indebted to Herman Hauschildt for performing our polishing experiments, creatively solving a host of fixturing problems, and dealing with the headaches of a pressurized slurry system. Blaine Beith, Hans Hansen, and Stan Edson provided the clever design and fabrication of the orbital polishing head. Lyle Weaver offered numerous insights into the convolution modeling. Kay T. Lu and Mark Zeleznock (summer intern) carried out the engineering of the high pressure slurry supply system. Thanks are extended to Mary Jessup for her help in typing.

\section{Notes and References}

\footnotetext{
${ }^{1}$ Report on the US Optics Market, Mitre Corporation, prepared for the Center for Optics Manufacturing (COM) and the American Precision Optics Manufacturers Association (APOMA), Rochester, NY, July 1993; the world-wide market for optical systems is projected to grow from $\$ 30 B$ in 1990 to $\$ 350 B$ in 2030; similarly, the world-wide market for optical components alone is projected to grow from $\$ 8.3 \mathrm{~B}$ in 1990 to $\$ 97 \mathrm{~B}$ in 2030.

${ }^{2}$ Zernike, F., "Specifications and metrology of surface figure and finish for microlithography optics", OSA TOPS on Extreme Ultraviolet Lithography, 1996, Vol. 4, Glenn D. Kubiak and Don Kania, (eds.) pp. 94-97.

${ }^{3}$ Carbone, F. A. and Markle, D., "Combining ion figuring and SPSI testing to produce a high quality, aspheric, 18" diameter, f/2 mirror", from Optical Manufacturing and Testing, SPIE vol. 2536, July 1995, pp. 89-98.

${ }^{4}$ Bollinger, L. D. and Zarowin, C. B., "Rapid, nonmechanical, damage-free figuring of optical surfaces using plasma-assisted chemical etching (PACE): Part I Experimental results", from Advances in Fabrication and Metrology for Optics and Large Optics, SPIE vol. 966, Aug. 1988, pp. 82-90. Also see two other papers on PACE in this volume.

${ }^{5}$ Allen, L. N., Keim, R. E., Lewis, T. S., and Ullom, J. R., "Surface error correction of a Keck 10-m telescope primary mirror segment by ion figuring", from Advanced Optical Manufacturing and Testing II, SPIE vol. 1531, July 1991, pp. 195-204.

"Namba, Y., Abe, M., "Ultraprecision grinding of optical glasses to produce super-smooth surfaces", Annals of the CIRP, 42(1), pp. 417-420 (1993).
} 
7 Blaedel, K. L. and Davis, P. J., "Evaluating a Glass Surface Produced by Ductile Grinding," Proceedings of the American Society for Precision Engineering - Subsurface Damage in Glass, 25 Apr 1989, Tucson AZ.

'Anderson, D. S., Angel, J. R., et. al., "Stressed-lap polishing of 3.5-m f/1.5 and 1.8-m f/1.0 mirrors", from Advanced Optical Manufacturing and Testing II, SPIE vol. 1531, July 1991, pp. 260-269.

${ }^{9}$ Lubliner, J. and Nelson, J. E., "Stressed mirror polishing. 1: A technique for producing non-axisymmetric mirrors", Applied Optics, 19(14), 15 July 1980, 2332-2340; Nelson, J. E., et. al., "Stressed mirror polishing. 2: Fabrication of an off-axis section of a paraboloid", Applied Optics, 19(14), 15 July 1980, 2341-2352.

${ }^{10}$ Mori, Y., et. al., "Evaluation of Elastic Emission Machined Surfaces by Scanning Tunneling Microscopy", J. Vac. Sci. \& Tech. A, 8(1), 621-624 (1990).

${ }^{11} \mathrm{Su}, \mathrm{Y}$., Wang, S., Chao, P., Hwang, Y., and Hsiau, J., "Investigation of elastic emission machining process: lubrication effects", Precision Engineering, vol. 17, no. 3, July 1995, pp. 164-172.

${ }_{12}$ Baker, P. C., "Advanced Flow-Polishing of Exotic Optical Materials", from X-Ray/EUV Optics for Astronomy and Microscopy, SPIE vol. 1160, 263-270 (1989).

${ }^{13}$ Bennett, J. M., Shaffer, J. J., Shibano, Y., and Namba, Y., "Float Polishing of Optical Materials", Applied Optics, 26(4), 696-703 (1987).

${ }^{14} \mathrm{Namba}, \mathrm{Y}$. and Tsuwa, H., "Float polishing of $\mathrm{MnZn}$ ferrites and characteristics of finished surfaces", in Proc. of the Fourth Int' Conference on Production Engineering, Japan Society for Precision Engineering and Int'l Institution for Production Engineering Research, Tokyo, 1980 pp. 1017-1022; this is one of several early papers on float polishing.

${ }^{15}$ Weis, O., "Direct contact polishing of sapphire", Applied Optics, vol. 31, no. 22, 1 Aug. 1992, pp. 4355-4362; this paper is not about float polishing per se, but discusses several relevant issues.

${ }^{16}$ Advanced Material Processing and Machining Technology Research Association (AAMTRA),

"Advanced Material Processing \& Machining System: Unveiling the Technology of the 21st Century", brochure describing the consortium's approach for soft $x$-ray lithography, members include Canon, Toshiba, Nikon, Hitachi, etc., c. 1991; note that some recent observations indicate that Canon may be expanding their approach for AAMTRA polishing techniques beyond EEM.

${ }_{17}$ The design of this tool has been awarded U. S. Patent No. 5,591,068.

${ }^{18}$ Moore Special Tool Company, Bridgeport, CT.

19 The theory of calculating shear stress are described by Schlichting, H., Boundary-Layer Theory, 7th edition, McGraw-Hill, San Francisco (1979).

${ }^{20}$ For a discussion of convolution, see McGillem, C. D. and Cooper, G. R., Continuous and Discrete Signal and System Analysis, Holt, Rinehart, and Winston, New York, second edition, 1984.

${ }^{21}$ For an application of convolution modeling to ion beam figuring, see Drueding, T. W., Bifano, T. G., and Fawcett, S. C., "Contouring algorithm for ion figuring", Precision Engineering, vol. 17, no., 1, Jan. 1995, pp. 10-21; and Granade, S. R. and Drueding, T. W., "Variation in material removal in Gaussian removal processes," Opt. Eng., 35(11), pp. 3267-3269 (November 1996).

${ }^{22}$ MATLAB is a software package and trademark of The Mathworks, Inc., Natick, Mass.

${ }^{23}$ The Surfanalyzer 1200 is a product of Federal Products Corp., Providence, RI.

${ }^{24}$ The NT-2000 white light interferometric microscope is a product of the WYKO Corp., Tucson, AZ

${ }^{25}$ The NewView is a product of the Zygo Corp., Middlefield, CT. 


\section{Appendix A Description of Polishing Testbed Numerical Control Interface}

The goal of the numerical control routine is to provide a convenient means for the machine operator to generate the commands for initiating machine motions or other control functions. The interface routine converts the commands to the specific language understood by the computer-numeric-control $(\mathrm{CNC})$ board. The $\mathrm{CNC}$ interprets these commands and generates the appropriate voltages to be supplied to the servo-amplifiers, which supply power to the appropriate motors. Position feedback from encoders (rotary and linear scales) is returned to the $\mathrm{CNC}$, thus offering closed-loop control. The CNC controller board used in this project is a Galil DMC 630 installed on a $90 \mathrm{MHz}$ Pentium computer system. The software was written in C under the National Instruments LabWindows/CVI development environment by Nhan Nguyen (LLNL).

This appendix describes the key interface routines that were prepared for this project's polishing testbed. The interface routines are used for several tasks:

- manually "moving" the machine for set up

- to program a series of sequential motions for simple polishing routines

- for calling up motion commands generated by other programs

- for saving motion commands

- for setting parameters for the CNC controller board.

Figure A-1 shows a window where a coordinated series of commands can be created. Using the INSERT button, a series of $x, y$ coordinates can be entered, each associated with a speed which is used for moving from that point. The coordinates and speeds are entered in the boxes in the upper left hand corner. The user has the option of referencing these coordinates in either a relative or an absolute sense, where the absolute coordinates are referenced according to a previously defined home position. As the list is created, the sequential commands are listed in the window at the lower right, and a pictorial is created in the lower left hand window to give the viewer a sense of the shape of the motion. The scaling in the pictorial window is automatically full-scale in both directions and may not be equal in $x$ and y. For example, the program shown in Figure A-1 is used to polish three equallyspaced square regions. Note that the SAVE and GET LIST buttons enable programs (lists of points, speeds) to be saved and recalled. For a complicated routine involving many points and differing velocities, the list can be generated using another program, such as a deconvolution routine used to calculate the required dwell times for removing a particular error profile.

A key aspect of computer-controlled polishing is the ability to vary the dwell time that the tool remains at any one location. This is practically accomplished by varying the traverse velocity of the tool, which scales as the inverse of the dwell 
time. Our polishing control routine allows both ramped and step-wise changes in speed. Various accelerations are created by inserting a number of points and approximating the desired acceleration with a series of ramped speed changes. A future version of the program will enable s-shaped accelerations between adjacent points.

Figure A-2 shows the control window used for running a pre-programmed routine, such as one created using the window shown in Figure A-1. The windows at the top show the current readout of the position feedback and the current speed as sensed by a velocity sensor, e.g. tachometer. The two indicators under the $x$ and $y$ readouts show the instantaneous error between the predicted (commanded) positions and the measured positions. The row of indicators labeled 'Next' are the next commanded coordinates and speed from the control program. The buttons at the right are used for starting, stopping, pausing, and continuing the routines. As the routine progresses, the pictorial window shows progress as a change in color superimposed on the planned motion path.

The Manual Control window in Figure A-3 shows the screen used for making single real-time machine motions called jogs. The current position and error indicators are shown at the top, as in Figure A-2. New positions and speeds can be entered below in either absolute or relative modes. The absolute coordinate home can be established simply by entering the coordinates of the current machine location in absolute mode and using the SET button(s). For moves involving both the $x$ and $y$ axes, the operator has the option of setting the speeds of each axis separately or by setting the vector speed. In the former case, the axes will generally not reach their endpoints at the same time, while in the vector mode, the both axes reach their conclusion at the same time. The vector mode is engaged by using the lower selection button located next to "Speed", by setting the speed in the single speed input box, and by using the GO XY button.

Also in the Manual Control window are icons for buttons and knobs that might be physically located on a manual control pedestal. The two rotary knobs are used for selecting among several pre-set jog distances and speeds; other values can be entered directly into the boxes at the left. The IN, OUT, LEFT, RIGHT, and STOP buttons are used for invoking the jog commands. A GO HOME button is provided for conveniently returning the machine to a predefined home position, such as might be used for part installation. The power to the motor can be switched ON/OFF using the MOTOR switch.

Figure A-4 shows a screen that is used for transmitting control parameters to the Galil computer control board. These include gains and values for the maximum accelerations that will be used. [Note that the values in this screen were chosen because we employ a specialized version of an LLNL-built servo-amplifier.] More information on the definition of these parameters is located in the Galil DMC 630 manual. 


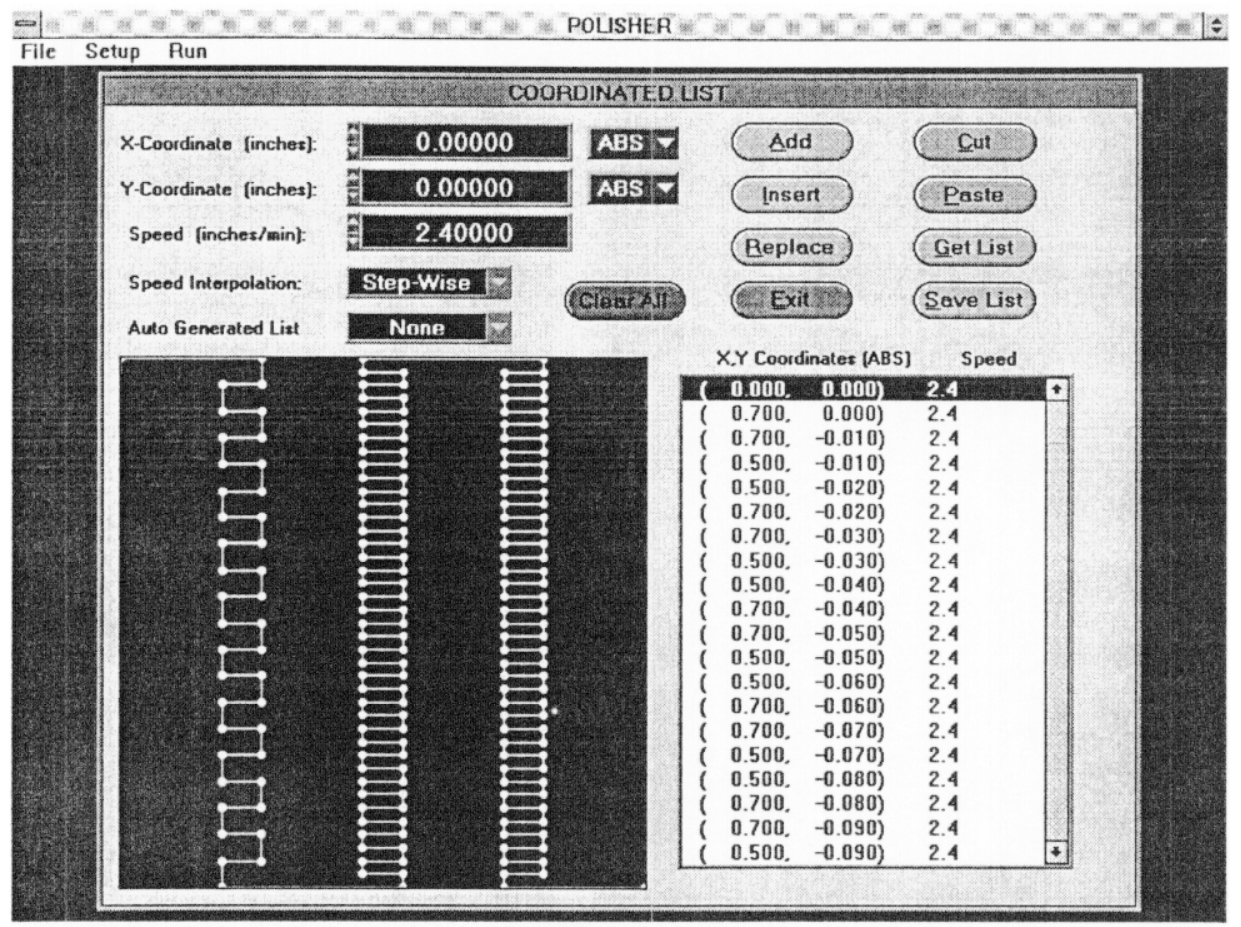

Figure A-1. CNC interface window for creating a coordinated list of machine motions with specified feedrates between points.

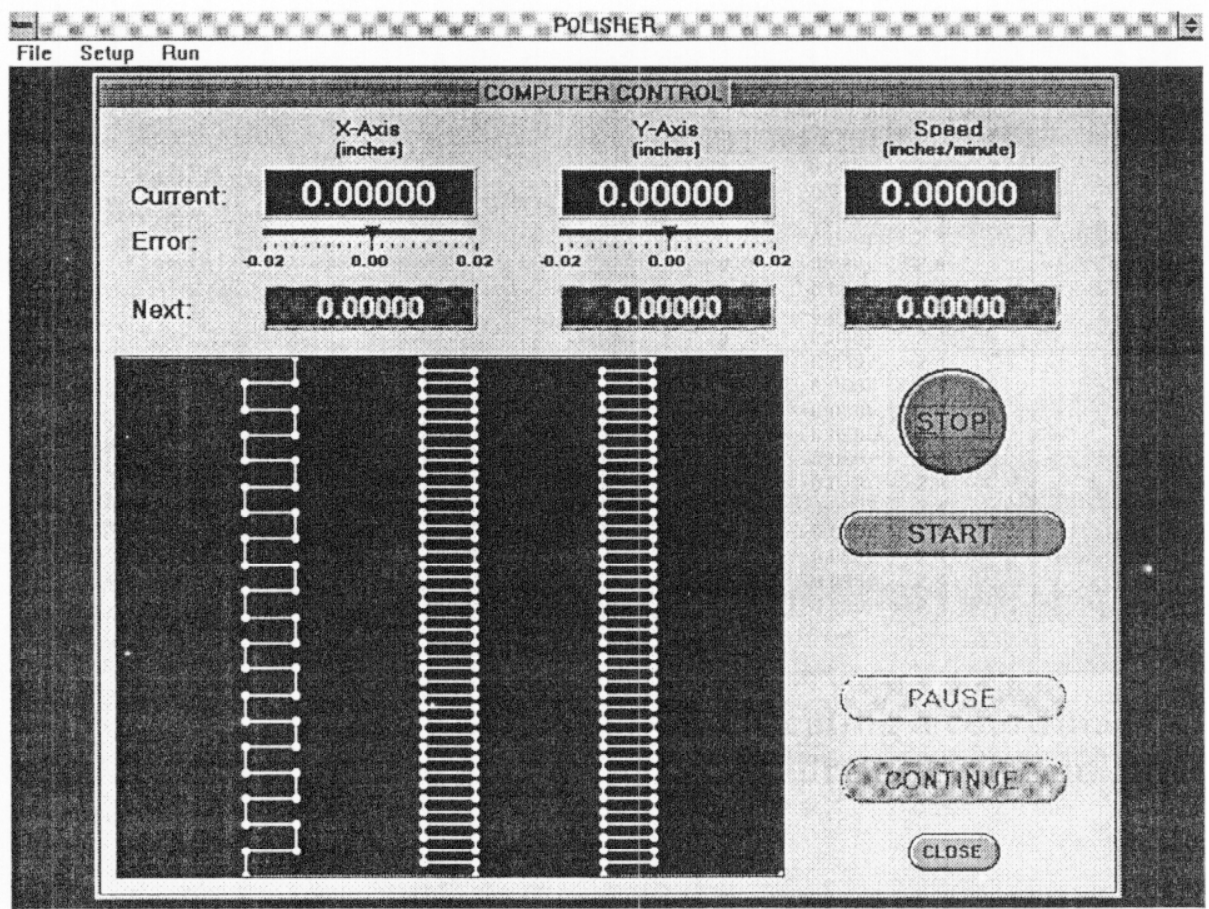

Figure A-2. Computer control window for initiating and ending a programmed polishing operation. 


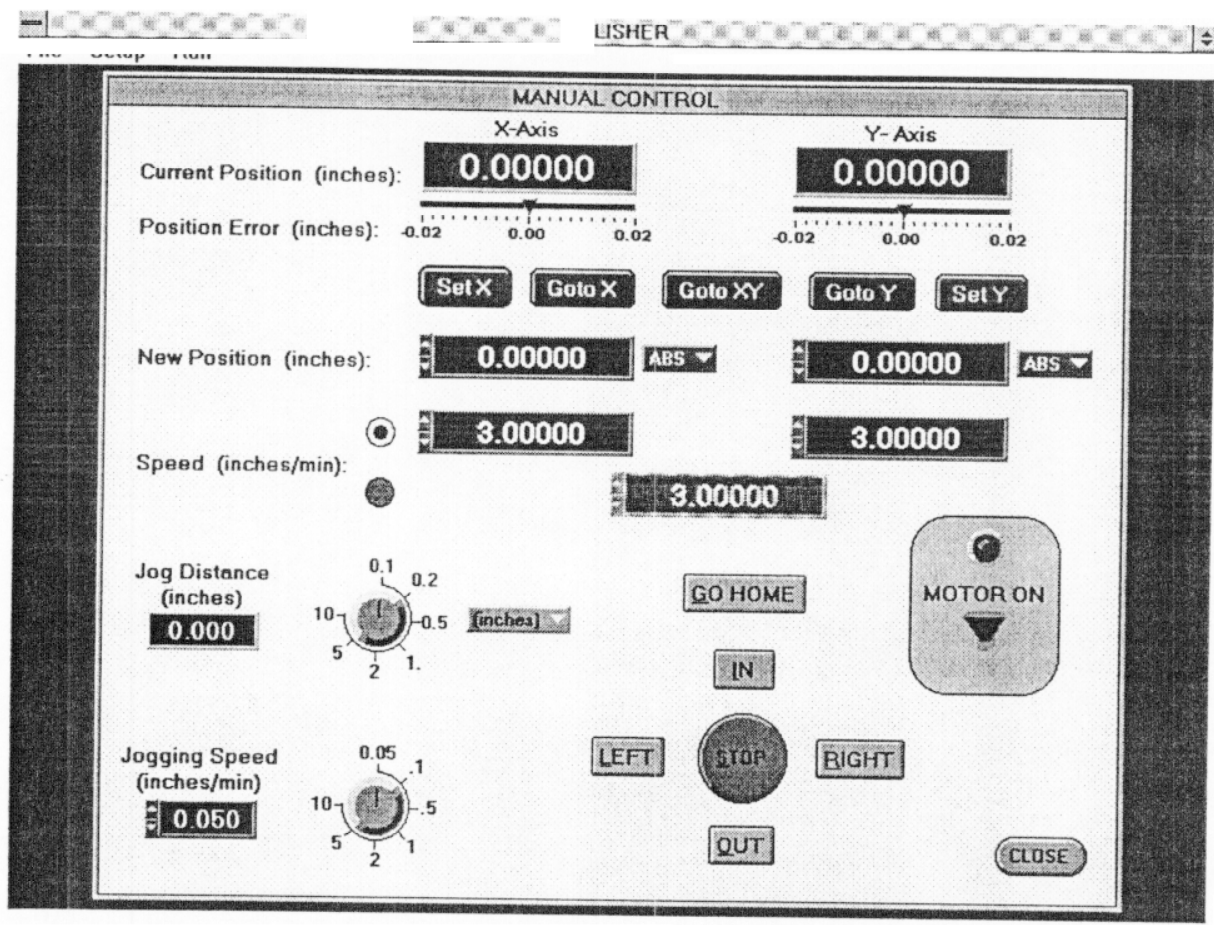

Figure A-3. Control panel for instantaneous machine motions.

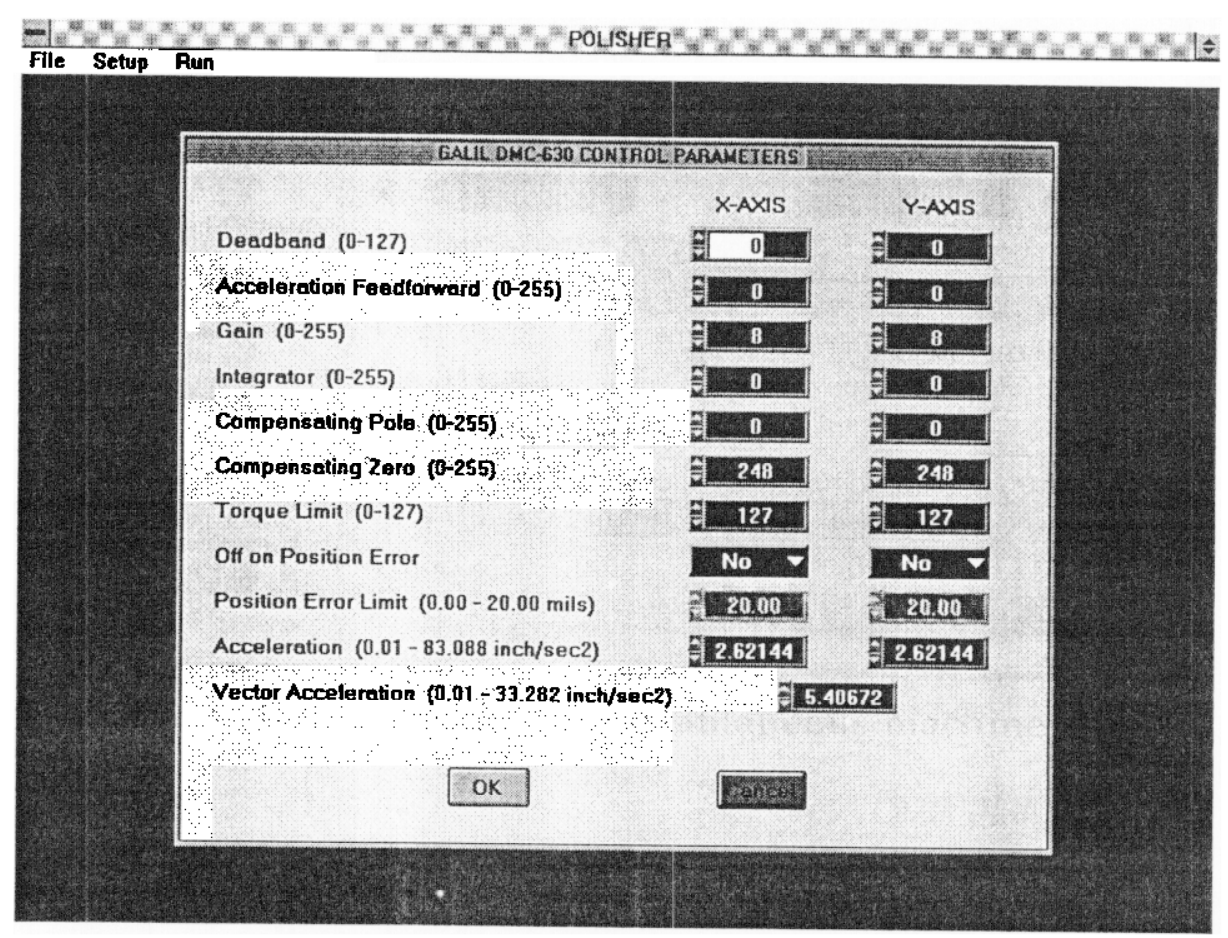

Figure A-4. Window for setting control parameters for the CNC controller board. 


\section{Appendix B: Derivation of Wall Shear Stress}

The diagram below outlines the modeling approach for calculating the shear stress distribution between the workpiece and the rotating polishing tool.

1. Formulate boundary layer equations for axisymmetric incompressible radial flow (Can be solved for shear stress)

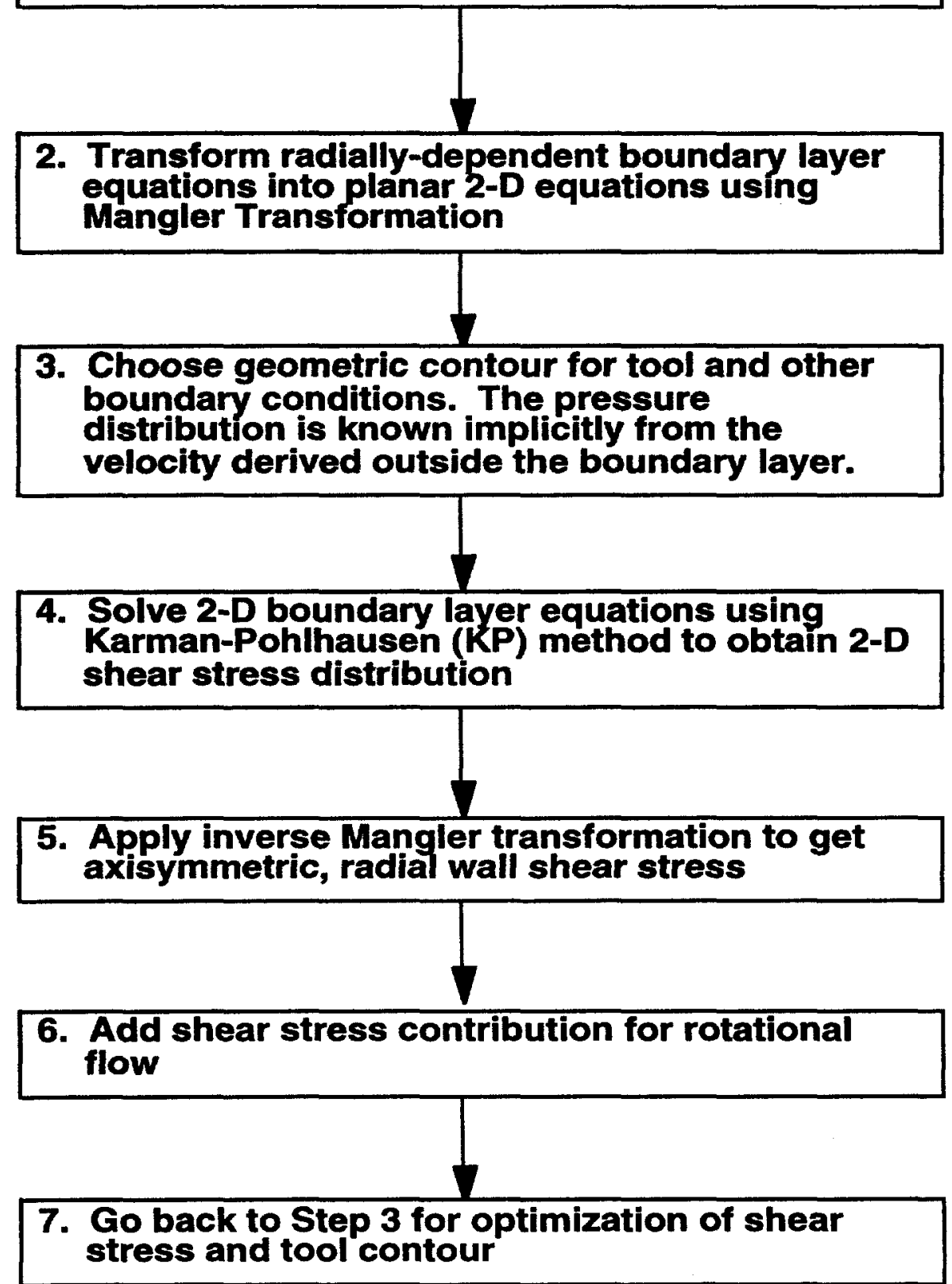

The derivation of the system's governing equations corresponds to the outlined model flow shown in Figure 1 and are the basic equations are referenced from "Boundary Layer Theory," by Schlichting'. 
1. We begin by formulating the boundary layer equations for an axisymmetric, incompressible radial flow [1].

$$
\begin{aligned}
& u \frac{\partial u}{\partial x}+v \frac{\partial u}{\partial y}=\frac{-1}{\rho} \frac{\partial p}{\partial x}+v \frac{\partial^{2} u}{\partial y^{2}} \\
& \frac{\partial u}{\partial x}+\frac{u}{x}+\frac{\partial v}{\partial y}=0
\end{aligned}
$$

where, $\mathbf{x}=0$ is centerline of axisymmetric system.

\section{Boundary Conditions:}

1. No slip at boundary layer and wall interface.

2. Edge of boundary layer approaches free stream velocity.

The free stream velocity will be estimated using a potential flow (inviscid) case to correspond with the second boundary condition. $U(x)$ is the average axial velocity taken to be only a function of $x, H(x)$ is the height of the flow passage and $m$ is the average flow rate.

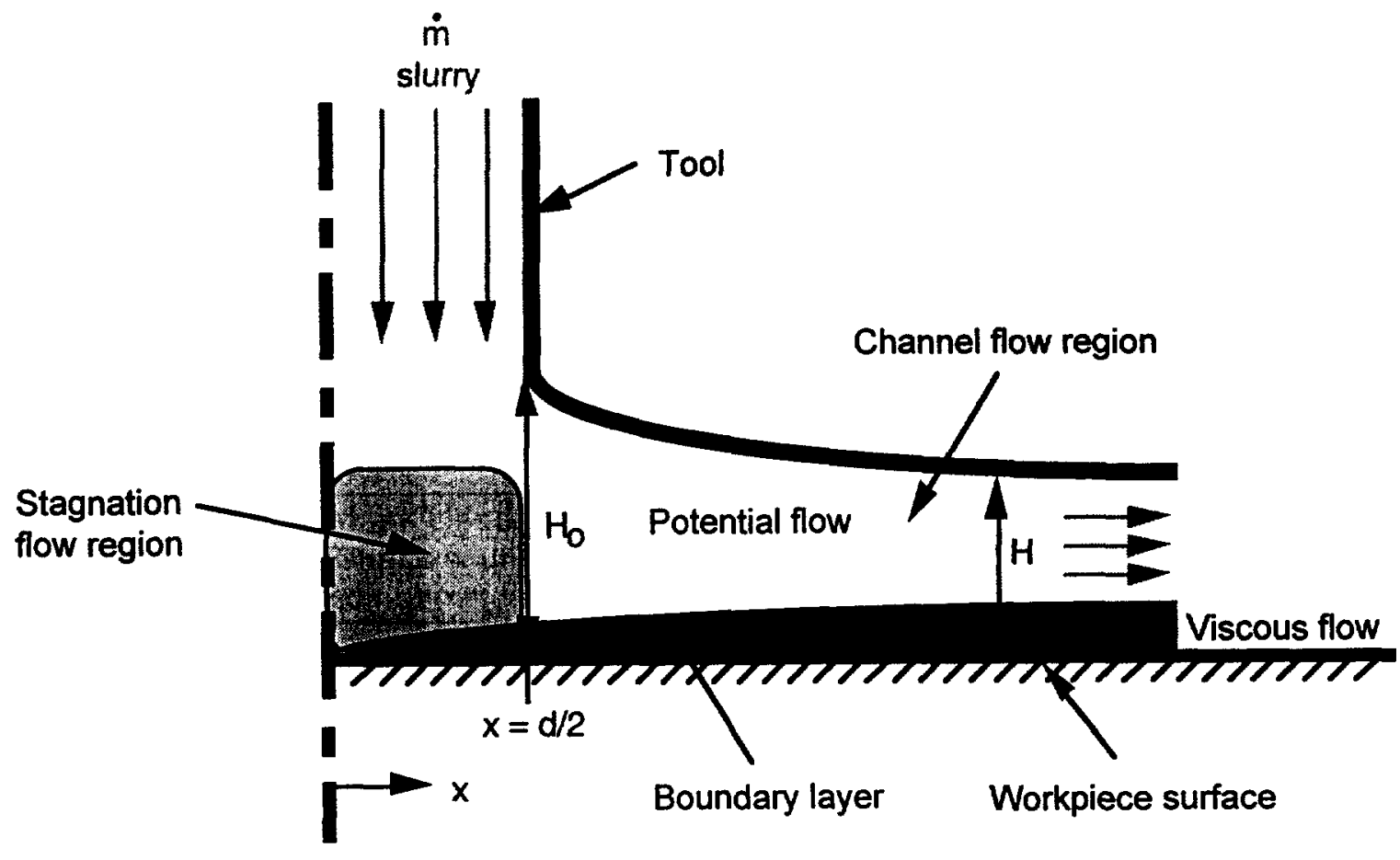

Centerline

Figure B-1. Schematic of the flow region between the workpiece and the rotating tool. Note that the shear stress $\tau_{w}$ is generated by the viscous flow inside the boundary layer.

The solution to the boundary layer equation depends on the velocity distribution outside it in the potential flow region. Refer to Figure 1 where the stagnation and channel flow regions are shown. The first region applies to small values of $\mathrm{x}$ and is the stagnation 
region. The second region applies to the developing channel flow and is applicable for larger values of $x$. In boundary layer theory, the boundary layer thickness is small compared to the potential flow regions outside it. In the potential flow region near the stagnation point

$$
\mathrm{U}(\mathrm{x})=\mathrm{a}_{1} \mathrm{x} \quad \text { small } \mathrm{x}
$$

while in the channel flow region

$$
\mathrm{U}(\mathrm{x})=\frac{\mathrm{a}_{2}}{\mathrm{xH}(\mathrm{x})} \quad \text { large } \mathrm{x}
$$

where $U(x)$ is the velocity outside the boundary layer. Simple mass balances (continuity) give the constants $a_{1}$ and $a_{2}$ as

$$
\begin{aligned}
& a_{1}=\frac{4 \dot{m}}{\pi \rho d^{2}} \\
& a_{2}=\frac{\dot{m}}{2 \pi \rho}
\end{aligned}
$$

where $\dot{m}$ is the total flow rate entering (and leaving).

Outside the boundary layer, equation 1 gives the potential flow $U(x)$ as:

$$
\mathrm{U} \frac{\partial \mathrm{U}}{\partial \mathrm{x}}=\frac{-1}{\rho} \frac{\partial \mathrm{p}}{\partial \mathrm{x}}
$$

thus, $\quad \frac{1}{2} \frac{\partial U^{2}}{\partial x}=\frac{-1}{\rho} \frac{\partial p}{\partial x}$

and integrating gives: $\quad \frac{p}{\rho}+\frac{U^{2}}{2}=$ constant

which is a form of Bernoulli's equation. Equations 3 and 4 will be used for the potential flow model. In boundary layer theory, pressure $\mathrm{p}$ is only a function of $\mathrm{x}$ (not $\mathrm{y}$ ).

2. Next we use the Mangler Transformation to transform radially-dependent boundary layer equations into 2-D planar equations [1,pg. 245].

The Mangler Transformation can be used to put the axisymmetric equations into a 2-D system with $\bar{x}, \bar{y}, \bar{U}$. The purpose of this is to be able to use the Karman-Pohlhausen solution methods applicable to $2-\mathrm{D}$ flows. If $\mathrm{x}$ and $\mathrm{y}$ are the axisymmetric variables, they transform into their two-dimensional, planar counterparts according to:

$$
\begin{aligned}
& \bar{x}=\frac{x^{3}}{3 L^{2}} \quad x=\left(3 L^{2} \bar{x}\right)^{1 / 3} \quad \text { ( } L \text { is a length scale) } \\
& \bar{y}=\frac{x}{L} y \quad y=\frac{L}{x} \bar{y}
\end{aligned}
$$




$$
\bar{u}=u \quad \bar{v}=\frac{L}{x}\left(v+\frac{1}{x} y u\right)
$$

Combine (1), (2), and (6b)

$\overline{\mathrm{u}} \frac{\partial \overline{\mathrm{u}}}{\partial \overline{\mathrm{x}}}+\overline{\mathrm{v}} \frac{\partial \overline{\mathrm{u}}}{\partial \overline{\mathrm{y}}}=\overline{\mathrm{U}} \frac{\mathrm{d} \overline{\mathrm{U}}}{\mathrm{d} \overline{\mathrm{x}}}+v \frac{\partial^{2} \overline{\mathrm{u}}}{\partial \overline{\mathrm{y}}^{2}}$

$\frac{\partial \bar{u}}{\partial \bar{x}}+\frac{\partial \bar{v}}{\partial \bar{y}}=0$

The 2-D transformed shear stress at the wall is given as

$$
\bar{\tau}_{\mathrm{w}}=\mu\left(\frac{\partial \overline{\mathrm{u}}}{\partial \overline{\mathrm{y}}}\right)_{0}
$$

Determine the relationship between $\tau_{w}$ (axisymmetric) and $\bar{\tau}_{w}$ (2D)

$$
\tau_{w}=\mu\left(\frac{\partial u}{\partial y}\right)_{y=0}=\mu\left(\frac{\partial \bar{u}}{\partial \bar{y}}\right)_{\bar{y}=0} \cdot\left(\frac{\partial u}{\partial \bar{u}}\right)\left(\frac{\partial \bar{y}}{\partial y}\right)=\frac{x}{L} \mu\left(\frac{\partial \bar{u}}{\partial \bar{y}}\right)_{\bar{y}=0}
$$

therefore,

$\tau_{w}=\frac{x}{L} \bar{\tau}_{w}$

Apply the Karman-Pohlhausen approximate solution for 2-D flows [1, pg. 215]

$\frac{\overline{\mathrm{U}} \theta^{2}}{v}=\frac{0.470}{\overline{\mathrm{U}}^{5}} \int_{\overline{\mathrm{x}}=0}^{\overline{\mathrm{x}}} \overline{\mathrm{U}}^{5} \mathrm{~d} \overline{\mathrm{x}}$

Where $\theta$ is the momentum thickness defined by:

$\theta \overline{\mathrm{U}}^{2}=\int_{0}^{\overline{\mathrm{y}}} \overline{\mathrm{u}}(\overline{\mathrm{U}}-\overline{\mathrm{u}}) d \overline{\mathrm{y}}$

Define a first shape factor, $Z$, given as,

$Z=\frac{\theta^{2}}{v}=\frac{0.470}{\bar{U}^{6}} \int_{0}^{\bar{x}} \bar{U}^{5} d \bar{x}$ 
Define a second shape factor as:

$$
K=Z \frac{d \bar{U}}{d \bar{x}}
$$

The wall shear stress is given as [1, eq. 10.32]

$$
\bar{\tau}_{w}=\frac{\mu \overline{\mathrm{U}}}{\theta} \mathrm{f}_{2}(\mathrm{~K})
$$

The relation between $f_{2}$ and $K$ has been numerically calculated by Holstein and Bohlen ${ }^{2}$ and is tabulated in Table 10.2 in reference 1.

3. Choose a geometric contour for tool and other boundary conditions. The pressure distribution is known implicitly from the velocity derived outside the boundary layer.

The model for the tool's flow field is divided into a stagnation region $(x<d / 2)$ and a channel flow of $(x>d / 2)$.

For our example, the contour for the channel flow is given as a function of $\mathbf{x}$.

$$
H(x)=H\left[\left(3 L^{2} \bar{x}\right)^{1 / 3}\right]=\bar{H}(\bar{x})
$$

where $H(x)$ is the height of the flow channel at position $x$.

4. Solve 2-D boundary layer equations using Karman-Pohlhausen method to obtain 2-D shear stress distribution.

We apply the Karman-Pohlhausen method as an approximate solution for the 2-D boundary layer equations [1]. First we divide the problem into two separate flow regions, one for $\mathrm{x}$ in the stagnation region and one for $\mathrm{x}$ in the channel flow region.

$$
\begin{array}{ll}
\text { small } x & x<d / 2 \\
\text { large } x & x>d / 2
\end{array}
$$

(a.) For small $x \quad U=\bar{U}=a_{1} x=a_{1}\left(3 L^{2}\right)^{1 / 3}(\bar{x})^{1 / 3}$

which gives $\quad \frac{d \bar{U}}{d \bar{x}}=\frac{a_{1}}{3}\left(3 L^{2}\right)^{1 / 3}(\bar{x})^{-2 / 3}$ 


$$
\begin{aligned}
Z & =\frac{0.470 a_{1}^{5}\left(3 L^{2}\right)^{5 / 3}}{a_{1}^{6}\left(3 L^{2}\right)^{2} \bar{x}^{2}} \int_{0}^{\bar{x}}(\bar{x})^{5 / 3} d \bar{x} \\
& =\frac{0.470}{a_{1}\left(3 L^{2}\right)^{1 / 3} \bar{x}^{2}}\left(\frac{3}{8}\right) \bar{x}^{8 / 3}=\left(\frac{3}{8}\right) \frac{0.470}{a_{1}\left(3 L^{2}\right)^{1 / 3}} \bar{x}^{2 / 3} \\
& =\left(\frac{3}{8}\right) \frac{0.470 \bar{x}}{a_{1}\left(3 L^{2} \bar{x}\right)^{1 / 3}}
\end{aligned}
$$

Now plugging (22) and (21) into (17) gives

$$
\begin{aligned}
K & =Z \frac{d \bar{U}}{d \bar{x}}=\left(\frac{3}{8}\right) \frac{0.470 \bar{x}}{a_{1}\left(3 L^{2} \bar{x}\right)^{1 / 3}} \frac{a_{1}}{3}\left(3 L^{2}\right)^{1 / 3} \bar{x}^{-2 / 3} \\
& =\frac{0.470}{8}=0.0587
\end{aligned}
$$

Using table 10.2 from reference $1: \quad f_{2}(0.0587)=0.305$

$$
\begin{aligned}
\mathrm{f}_{2} & =\frac{\theta \bar{\tau}_{\mathrm{w}}}{\mu \overline{\mathrm{U}}} \\
\theta & =\sqrt{\mathrm{Zv}} \\
\bar{\tau}_{\mathrm{w}} & =\frac{\mu \overline{\mathrm{U}}}{\sqrt{\mathrm{Zv}}} \cdot \mathrm{f}_{2} \\
\bar{\tau}_{\mathrm{w}} & =\frac{\mu \mathrm{a}_{1}\left(3 \mathrm{~L}^{2}\right)^{1 / 3} \overline{\mathrm{x}}^{1 / 3}(0.305)}{\sqrt{v}\left(\frac{3}{8} \frac{0.470 \overline{\mathrm{x}}}{\mathrm{a}_{1}}\right)^{1 / 2}\left(3 \mathrm{~L}^{2} \overline{\mathrm{x}}\right)^{-1 / 6}} \\
& =\left(\frac{8 \mathrm{a}_{1}^{3}}{0.470 v}\right)^{1 / 2}(0.305) \mu \mathrm{L}
\end{aligned}
$$


(b.) For large $x$ where $\quad x>\frac{d}{2} \rightarrow \bar{x}>\frac{(d / 2)^{3}}{3 L^{2}}$

$$
\text { define } m=\frac{(d / 2)^{3}}{3 L^{2}}
$$

Include contributions for both small $\mathrm{x}$ and large $\mathrm{x}$. The integral is defined from $\mathrm{x}=\mathrm{zero}$, the large $x$ region begins at $x=d / 2$.

$Z=\frac{0.470}{\bar{U}^{6}}\left[\int_{0}^{m} \bar{U}^{s} d \bar{x}+\int_{m}^{\bar{x}} \bar{U}^{s} d \bar{x}\right]$

Using $\bar{U}=a_{1}\left(3 L^{2}\right)^{1 / 3} \bar{x}^{1 / 3} \quad$ for small $x$

$$
\bar{U}=\frac{a_{2} \bar{x}^{-1 / 3}}{\left(3 L^{2}\right)^{1 / 3} \bar{H}(\bar{x})} \quad \text { for large } x
$$

$$
\begin{aligned}
Z & =\frac{0.470}{\bar{U}^{6}}\left[a_{1}^{5}\left(3 L^{2}\right)^{5 / 3} \int_{0}^{m} \bar{x} d \bar{x}+a_{2}^{5} \int_{0}^{m} \frac{\left(3 L^{2} \bar{x}\right)^{-5 / 3}}{\bar{H}(\bar{x})^{5}} d \bar{x}\right] \\
& =\frac{0.470}{\bar{U}^{6}}\left[a_{1}^{5}\left(3 L^{2}\right)^{5 / 3}\left(\frac{3}{8}\right) m^{8 / 3}+\left(\frac{a_{2}}{\left(3 L^{2}\right)^{1 / 3}}\right)^{5} \frac{\bar{x}}{\bar{H}(\bar{x})^{5}} d \bar{x}\right] \\
& =\frac{0.470}{\bar{U}^{6}}\left[a_{1}^{5}\left(3 L^{2}\right)^{5 / 3}\left(\frac{3}{8}\right)\left(\frac{d}{2}\right)^{8}\left(3 L^{2}\right)^{-8 / 3}+\left(\frac{a_{2}}{\left(3 L^{2}\right)^{1 / 3}}\right)^{5} \frac{\bar{x}}{\overline{\mathrm{H}}(\bar{x})^{5}} d \bar{x}\right] \\
& =\frac{0.470}{\bar{U}^{6}}\left[\frac{a_{1}^{5}\left(\frac{d}{2}\right)^{8}}{8 L^{2}}+\left(\frac{a_{2}}{\left(3 L^{2}\right)^{1 / 3}}\right)^{5} \frac{\bar{x}}{\overline{\mathrm{x}}(\bar{x})^{5}} d \bar{x}\right]
\end{aligned}
$$




$$
\begin{aligned}
\frac{d \bar{U}}{d \bar{x}} & =\frac{a_{2}}{\left(3 L^{2}\right)^{1 / 3}} \frac{\frac{-1}{3} \bar{x}^{-4 / 3} \bar{H}(\bar{x})-\overline{H^{\prime}}(\bar{x}) \bar{x}^{-1 / 3}}{\bar{H}(x)^{2}} \\
& =\frac{a_{2} \bar{x}^{-4 / 3}}{3\left(3 L^{2}\right)^{1 / 3} \bar{H}(x)}\left[1+\frac{3 \bar{x} \bar{H}^{\prime}(\bar{x})}{\bar{H}(\bar{x})}\right] \\
K & =Z \frac{d \bar{U}}{d \bar{x}} \\
& =\frac{0.470}{\bar{U}^{6}}\left[\frac{\left(\frac{d}{2}\right)^{8}}{8 L^{2}}+\left(\frac{a_{2}}{\left(3 L^{2}\right)^{1 / 3}}\right)^{5} \int_{m}^{\bar{x}} \frac{\bar{x}^{-5 / 3}}{\bar{H}(\bar{x})^{5}} d \bar{x}\right. \\
\bar{\tau}_{w} & =\frac{\mu \bar{U}}{\sqrt{Z v}} f_{2}(K)
\end{aligned}
$$

5. Apply the inverse Mangler transformation to get the axisymmetric radial wall shear stress

(a.) For small $\mathrm{x}$ :

Transform equation 23 using $\quad \bar{x}=\frac{x^{3}}{3 L^{2}} \quad d \bar{x}=\left(\frac{x}{L}\right)^{2} d x$ $\bar{\tau}_{\mathrm{w}}=\frac{\mathrm{x}}{\mathrm{L}} \bar{\tau}_{\mathrm{w}}=\frac{\mathrm{x}}{\mathrm{L}}\left(\frac{8 \mathrm{a}_{1}{ }^{3}}{0.470 \mathrm{v}}\right)^{1 / 2}(0.305) \mu \mathrm{L}=\left(\frac{8 \mu \rho \mathrm{a}_{1}{ }^{3}}{0.470}\right)^{1 / 2} 0.305 \mathrm{x}$

since $v=\frac{\mu}{\delta}$

Note that the length scale $\mathrm{L}$ does not appear, which is crucial. 
(b.) For large $x \quad \bar{x}>m \rightarrow x>\frac{d}{2}$

$\bar{x}=\frac{x^{3}}{3 L^{2}} \quad d \bar{x}=\left(\frac{x}{L}\right)^{2} d x$

$\tau_{\mathrm{w}}=\bar{\tau}_{\mathrm{w}} \frac{\mathrm{x}}{\mathrm{L}}=\frac{\mu \overline{\mathrm{U}}}{\sqrt{\mathrm{Zv}}} \frac{\mathrm{x}}{\mathrm{L}} \mathrm{f}_{2}(\mathrm{~K})$

$\overline{\mathrm{H}}(\overline{\mathrm{x}}) \rightarrow \mathrm{H}(\mathrm{x})$

$\bar{H}^{\prime}(\bar{x})=\frac{d \bar{H}(\bar{x})}{d \bar{x}}=\frac{d H(x)}{d x} \cdot \frac{d x}{d \bar{x}}=H(x) \frac{L^{2}}{x^{2}}$

$\overline{\mathrm{U}}=\frac{\mathrm{a}_{2}}{\left(3 \mathrm{~L}^{2}\right)^{1 / 3} \overline{\mathrm{H}}(\overline{\mathrm{x}})}=\mathrm{U}=\frac{\mathrm{a}_{2}}{\mathrm{xH}(\mathrm{x})}$

Transform $Z$ from equation 26

$$
\begin{aligned}
Z & =\frac{0.470 x^{6} H(x)^{6}}{a_{2}^{6}}\left[\frac{a_{1}^{5}\left(\frac{d}{2}\right)^{8}}{8 L^{2}}+\left(\frac{a_{2}}{\left(3 L^{2}\right)^{1 / 3}}\right)^{5} \int_{\frac{d}{2}}^{x} \frac{\left(\frac{x^{3}}{3 L^{2}}\right)^{-5 / 3}}{H(x)^{5}} \frac{x^{2}}{L^{2}} d x\right] \\
& =\frac{0.470}{L^{2}}\left(\frac{x H(x)}{a_{2}}\right)^{6}\left[\frac{a_{1}^{5}\left(\frac{d}{2}\right)^{8}}{8}+a_{2}^{5} \int_{\frac{d}{2}}^{\bar{x}} \frac{d x}{x^{3} H(x)^{5}}\right]
\end{aligned}
$$

Note that the dimension of $\mathrm{Z}$ is time. The transform of equation 27 :

$$
\begin{aligned}
\frac{d \bar{U}}{d \bar{x}} & =\frac{-a_{2}\left(\frac{x^{3}}{3 L^{2}}\right)^{-4 / 3}}{3\left(3 L^{2}\right)^{1 / 3} H(x)}\left[1+\frac{3\left(\frac{x^{3}}{3 L^{2}}\right) H(x) \frac{L^{2}}{x^{2}}}{H(x)}\right] \\
& =-\left(\frac{L}{x}\right)^{2}\left(\frac{a_{2}}{x^{2} H(x)}\right)\left(1+\frac{x H(x)}{H(x)}\right)
\end{aligned}
$$




$$
\begin{aligned}
K=Z \frac{d \bar{U}}{d \bar{x}}=-\left(\frac{L}{x}\right)^{2}\left(\frac{a_{2}}{x^{2} H(x)}\right)\left(1+\frac{x H(x)}{H(x)}\right) \\
\cdot \frac{0.470}{L^{2}}\left(\frac{x H(x)}{a_{2}}\right)^{6}\left[\frac{a_{1}^{5}\left(\frac{d}{2}\right)^{8}}{8}+a_{2}^{5} \int_{\frac{d}{2}}^{x} \frac{d x}{x^{3} H(x)^{5}}\right] \\
=\frac{-0.470 x^{2} H(x)^{5}}{a_{2}^{5}}\left(1+\frac{x H(x)}{H(x)}\right)\left[\frac{a_{1}^{5}\left(\frac{d}{2}\right)^{8}}{8}+a_{2}^{5} \int_{\frac{d}{2}}^{x} \frac{d x}{x^{3} H(x)^{5}}\right]
\end{aligned}
$$

Solve for the shear stress using equations 31,32 , and 33

$$
\begin{aligned}
\tau_{w} & =\frac{\mu U}{\sqrt{Z v}} \frac{x}{L} f_{2}(K) \\
& =\frac{\sqrt{\mu \rho} a_{2} f_{2}(K)}{H(x) L}\left[\frac{0.470}{L^{2}}\left(\frac{x H(x)}{a_{2}}\right)^{6}\left(\frac{a_{1}^{5}\left(\frac{d}{2}\right)^{8}}{8}+a_{2}^{5} \int_{\frac{d}{2}}^{x} \frac{d x}{x^{3} H(x)^{5}}\right]^{-1 / 2}\right. \\
& =\sqrt{\frac{\mu \rho}{0.470}}\left(\frac{a_{2}}{H(x)}\right)^{4} \frac{f_{2}(K)}{x^{3}}\left[\frac{a_{1}^{5}\left(\frac{d}{2}\right)^{8}}{8}+a_{2}^{5} \int_{\frac{d}{2}}^{x} \frac{d x}{x^{3} H(x)^{5}}\right]^{-1 / 2}
\end{aligned}
$$

$f_{2}(K)$ is found in Table 10.2 in ref. 1 using the value of $K$ calculated using equation 33.

6. Add shear stress contribution for rotational flow.

The contribution of the rotational flow to the shear stress is small relative to the that from the radial flow for many of the tools considered. It was not included in this current development, but will be important for tool geometries that emphasize rotation with minimal contributions from constrained jet flowfield. 


\section{Example}

We calculated the wall shear stress for a tool that contour in the channel region given by the following profiles:

$$
\begin{array}{ll}
\frac{H(x)}{H_{0}}=\exp \left[-2\left(\frac{2 x}{d}\right)-1.0348\left(\frac{2 x}{d}-1\right)^{2}\right] & \text { for } 1<\frac{2 x}{d} \leq 2.4 \\
\frac{H(x)}{H_{0}}=0.008+0.018\left(\frac{\frac{2 x}{2.4}}{d}\right)^{2} & \text { for } 2.4<\frac{2 x}{d} \leq 4
\end{array}
$$

The numerical results are plotted below, where the normalized shear stress is defined as:

$$
\tau_{\text {norm }} \equiv \frac{\tau_{\mathrm{w}}(\mathrm{x})}{\left(\frac{8 \mu \rho \mathrm{a}_{1}^{3}}{0.470}\right)^{1 / 2} \frac{\mathrm{d}}{2}}
$$

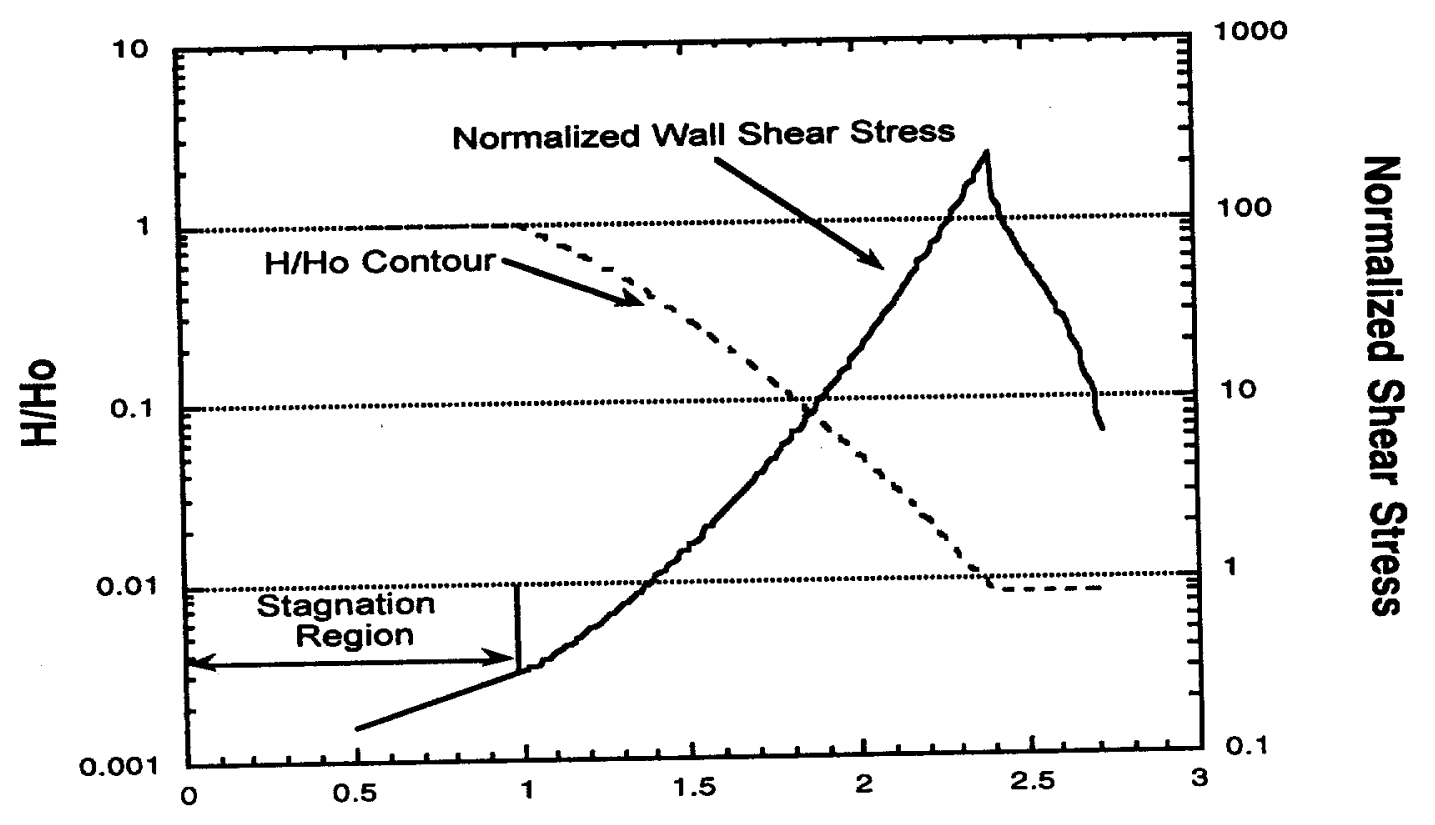




\section{Summary:}

$$
\begin{gathered}
\text { for } x<\frac{d}{2}: \\
\tau_{w}=\left(\frac{8 \mu \rho a_{1}^{3}}{0.470}\right)^{1 / 2} 0.305 x \\
\text { for } x>\frac{d}{2} \\
\tau_{w}=\sqrt{\frac{\mu \rho}{0.470}}\left(\frac{a_{2}}{H(x)}\right)^{4} \frac{f_{2}(K)}{x^{3}}\left[\frac{a_{1}^{5}\left(\frac{d}{2}\right)^{8}}{8}+a_{2}^{5} \int_{\frac{d}{2}}^{x} \frac{d x}{x^{3} H(x)^{5}}\right]^{-1 / 2}
\end{gathered}
$$

where

$$
K=\frac{-0.470 x^{2} H(x)^{5}}{a_{2}^{5}}\left(1+\frac{x H(x)}{H(x)}\left[\frac{a_{1}^{5}\left(\frac{d}{2}\right)^{8}}{8}+a_{2}^{5} \int_{\frac{d}{2}}^{x} \frac{d x}{x^{3} H(x)^{5}}\right]\right.
$$

and the numerical solution for $f_{2}(K)$ is located in Table 10.2 in reference 1 .

\footnotetext{
${ }^{1}$ Schlichting, H., Boundary Layer Theory, 7th Edition, McGraw-Hill, San Francisco (1979).

${ }^{2}$ Holstein, H. and Bohlen, T., "Ein einfaches Verfahren zur Berechnung laminarer Reibungsschichten, die dem Näherungsverfahren von K. Pohlhausen genügen", Lilenthal-Bericht S. 10, 5-16 (1940); the results used in this report were taken from reference 1 .
} 


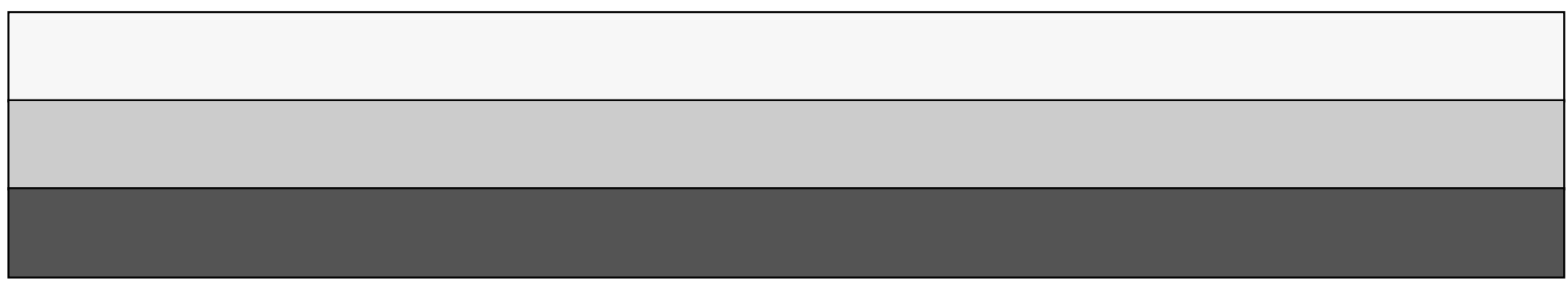

